\title{
High-throughput functional analysis of natural variants in yeast
}

$5 \quad{ }^{1}$ Department of Genome Sciences, University of Washington, Seattle, Washington 98195.

$6 \quad{ }^{2}$ Université de Strasbourg, CNRS, GMGM UMR 7156, Strasbourg, France

$7 \quad{ }^{3}$ Institut Universitaire de France (IUF), Paris, France

8

\section{Abstract}

How natural variation affects phenotype is difficult to determine given our incomplete ability to deduce the functional impact of the polymorphisms detected in a population. Although current computational and experimental tools can predict and measure allele function, there has previously been no assay that does so in a high-throughput manner while also representing haplotypes derived from wild populations. Here, we present such an assay that measures the fitness of hundreds of natural alleles of a given gene without site-directed mutagenesis or DNA synthesis. With a large collection of diverse Saccharomyces cerevisiae natural isolates, we piloted this technique using the gene $S U L 1$, which encodes a high-affinity sulfate permease that, at increased copy number, can improve the fitness of cells grown in sulfate-limited media. We cloned and barcoded all alleles from a collection of over 1000 natural isolates en masse and matched barcodes with their respective variants using PacBio long-read sequencing and a novel error-correction algorithm. We then transformed the reference S288C strain with this library and used barcode sequencing to track growth ability in sulfate limitation of lineages carrying each allele. We show that this approach allows us to measure the fitness conferred by each allele and stratify functional and nonfunctional alleles. Additionally, we pinpoint which polymorphisms in both coding and noncoding regions are detrimental to fitness or are of small 
27 effect and result in intermediate phenotypes. Integrating these results with a phylogenetic tree,

28 we observe how often loss-of-function occurs and whether or not there is an evolutionary

29 pattern to our observable phenotypic results. This approach is easily applicable to other genes.

30 Our results complement classic genotype-phenotype mapping strategies and demonstrate a

31 high-throughput approach for understanding the effects of polymorphisms across an entire

32 species which can greatly propel future investigations into quantitative traits.

\section{Background}

Quantitative traits, or traits that vary on a continuous distribution rather than in discrete categories, are responsible for most phenotypic differences across all organisms (MacKay et al., 2009; Morgante et al., 2018). Despite decades of efforts investigating how genotype informs phenotype, the molecular underpinnings of quantitative traits are still largely unknown, especially on a species-wide scale. Understanding how sequence changes lead to phenotypic changes is difficult to disentangle as these traits often involve interactions between multiple loci

41 that each in themselves have genetic variation among natural populations. Even for a single

42 locus, an exhaustive population-scale determination of genetic variants impacting phenotype

43 remains out of sight. Improved approaches for investigating this in a cost-effective and high-

44 throughput manner will greatly broaden insights into the genetic basis of trait variation, ranging

45 from deleterious diseases to adaptive evolution.

Due to the rapid advancement and decreased cost of high-throughput sequencing,

47 forward genetics approaches have boosted our ability to pinpoint loci underlying traits of

48 interest. For instance, quantitative trait loci (QTL) and linkage mapping provide avenues for

49 identifying loci responsible for phenotypic differences between individuals, and even in some

50 instances can result in determining what polymorphisms are integral for certain phenotypes

51 (Ehrenreich et al., 2012; Treusch et al., 2015). However, this method relies on pairwise crosses

52 between a small subset of genetic backgrounds and is difficult to scale to investigate phenotypic 
53 variation on the population level (Stinchcombe and Hoekstra, 2008). Approaches like genome-

54 wide association studies do investigate loci on the population scale, but lack the ability to infer

55 and experimentally functionalize the effects of rare or low-frequency variants (Morgante et al.,

56 2018). Other computational approaches deduce function based on collected data describing

57 metrics such as conservation, statistical analyses of genomic architecture, allele frequency,

58 predicted changes in protein stability, known sites of protein-protein interactions, and

59 transcription factor-binding motifs, but all still require experimental validations (Adzhubei et al.,

60 2010; Mitchell-Olds et al., 2007; Schymkowitz et al., 2005; She and Jarosz, 2018; Wagih et al.,

61 2018; Wray et al., 2013).

62 Recently, multiplexed assays of variant effects (MAVE) studies have provided an

63 approach for functionalizing thousands of variants in a high-throughput manner (Starita et al.,

64 2017; Weile and Roth, 2018). These have been extremely useful in understanding how

65 missense mutations or nucleotide changes alter gene function and/or expression (Duveau et al.,

66 2017; Fowler and Fields, 2014; Matreyek et al., 2018). However, most of these approaches

67 have been limited to studying single nucleotide or amino acid substitutions away from a

68 reference sequence, as technologies don't yet exist to generate and measure the

69 consequences of the large libraries that would be necessary to explore combinatorial variation.

70 Additionally, the majority of variants assayed are rarely reflective of those in natural populations.

71 For instance, natural alleles can have more than one polymorphism, not all of which are seen

72 exclusively in coding or exclusively in noncoding regions, and thus are not surveyed completely

73 in many MAVE studies. Being able to directly test the function of natural variants of whole

74 populations provides context for how polymorphisms and combinations of polymorphisms alter

75 phenotype. Furthermore, such an approach would provide deeper insight into the evolutionary

76 history of a gene and how both weak and strong selection or drift have acted upon a phenotype

77 that results in the variation present in natural populations (Johnson and Barton, 2005; Mitchell- 
78 Olds et al., 2007). Thus, developing a method for testing natural variants in a high-throughput

79 manner is of high interest.

Here, we developed such an assay functionalizing natural variants on a species-wide scale using Saccharomyces cerevisiae, the budding yeast. With the rapid advancement of highthroughput whole-genome sequencing, we now have large collections of natural $S$. cerevisiae strains that contain genomic data as well as geographical and ecological information (Bergström et al., 2014; Liti et al., 2009; Peter et al., 2018; Schacherer et al., 2009; Strope et al., 2015; Zhu et al., 2016). Although much research has been done on laboratory strains for understanding biology, curation of these collections revealed the striking diversity within this popular model organism: S. cerevisiae has been isolated from a variety of countries all over the globe and from habitats like human clinical samples, domesticated products like beer and bread, and tree and fruit samples. Sequencing of these genomes has revealed a lot about genetic variation, but still little is known about how these genetic changes impact phenotypic variation outside of a handful of association studies and QTL mapping efforts (Ehrenreich et al., 2012, 2009; Kim et al., 2012; Peltier et al., 2019; Wilkening et al., 2014). With the large genome sequencing efforts and strain collections, in addition to the wealth of molecular tools developed for yeast, $S$. cerevisiae is the

94 ideal system to develop this assay and investigate the effects of natural polymorphisms for 95 whole populations.

For piloting and developing our approach, we used the natural alleles of SUL1 from a

97 collection of 1,011 isolates to test whether we can deconvolute how variation affects cell growth

98 under sulfate limiting conditions. SUL1 encodes a high-affinity sulfate permease and is

99 expressed under sulfate limitation. Previous studies have found that when evolving different

100 strains of S. cerevisiae under sulfate limitation in the chemostat, cells with amplifications of

101 SUL1 have high fitness and rise in frequency in the population (Gresham et al., 2008; Payen et

102 al., 2014; Sanchez et al., 2017). Strong selection for amplification of this locus in sulfate-limiting

103 conditions allows for a reliable functional assay in which we can mimic amplifications by 
104 transforming cells with a low-copy plasmid containing SUL1. Additionally, we have previously

105 performed a deep mutational scan on the promoter of SUL1, giving us a dataset measuring the

106 functional consequences of single mutations for comparison (Rich et al., 2016). By co-culturing

107 a population of cells transformed with a barcoded library of natural alleles, we can measure

108 competitive fitness via barcode sequencing and thereby determine SUL 1 functionality en

109 masse. Our results show that this assay is accurate in predicting function and useful in

110 understanding what genetic changes affect phenotype. These data allow for insight into the

111 evolutionary history of SUL1 function and possible evidence for selection of loss-of-function

112 mutations. This approach, especially when combined with established forward genetics

113 approaches in identifying causal loci, will greatly strengthen our understanding of quantitative

114 traits on a species-wide scale.

Methods

Strains and plasmids

Natural isolates from the 1,011 Saccharomyces cerevisiae collection were used to

119 isolate natural variants of SUL1 (Peter et al., 2018). Strains pinned on yeast extract peptone

120 dextrose (YPD) agar plates were transferred to liquid YPD in 96-well plates, grown overnight at

$12130^{\circ} \mathrm{C}$, and stored in $30 \%$ glycerol at $-80^{\circ} \mathrm{C}$. The FY3 S288C strain DBY7284 (MATa ura3-52)

122 was used for transformation and competition experiments (described below). A GFP-marked

123 strain YMD1214 (MATa hoL::GFP-KANMX) that has neutral fitness under sulfate limitation was

124 used for validation competition assays. Prototrophic FY3 (DBY11069), YMD4321 (MATa ura3-

12552 sul14::URA3-KANMX), YMD4322 (MATa ura3-52 sul2A::URA3-KANMX), and YMD4323

126 (MATa ura3-52 sul14::URA3-KanMX sul2A::URA3-KanMX) were used to validate growth rates

127 on sulfate-limited and sulfate-abundant agar plates. A pRS316 vector with an Nrul site inserted

128 in the BamHI site (YMD2307) was used in this study for molecular cloning and competitions

129 described below. A complete list of strains can be found in Supplementary Table 1. 
Plasmid and yeast library generation

Strains from the $1,011 \mathrm{~S}$. cerevisiae collection were pooled together from colonies on a solid agar plate. Genomic DNA was then extracted using the QIAGEN Genomic-tip 100/G kit.

134 Natural variants of SUL1 were amplified with primers designed to hybridize to conserved regions 844 bp upstream of the translation start site and 262 bp downstream of the stop codon (oligos 1 and 2, (Supplementary Table 2)). Oligo 2 also contained an 8 bp randomized sequence to serve as a barcode. PCR was performed using KAPA HiFi Hotstart Readymix with

138 the following cycling conditions: $95^{\circ} \mathrm{C}$ for $3 \mathrm{~min}$, then 19 cycles of $98^{\circ} \mathrm{C}$ for 20 seconds, $60^{\circ} \mathrm{C}$ for 13915 seconds, and $72^{\circ} \mathrm{C}$ for 4 minutes. Final extension was at $72^{\circ} \mathrm{C}$ for 4 minutes, and then the 140 reaction was cooled to $4^{\circ} \mathrm{C}$. The barcoded product was purified using the DNA Clean and

141 Concentrator kit from Zymo Research and assembled into an Nrul-digested plasmid via Gibson

142 assembly. Chemically competent $E$. coli cells were transformed with the product using heat 143 shock at $42^{\circ} \mathrm{C}$, and $>20,000$ transformants were collected and pooled. Plasmids were extracted 144 from the pooled transformants using Wizard® Plus SV Miniprep DNA Purification Kit and then 145 used to transform yeast (DBY7284) using $100 \mu \mathrm{L}$ of $2 \mathrm{M}$ lithium acetate, $800 \mu \mathrm{L}$ of $50 \% 4000$ 146 polyethylene glycol, $100 \mu \mathrm{L}$ of $1 \mathrm{M}$ dithiothreitol, and $50 \mu \mathrm{L}$ of $10 \mathrm{mg} / \mathrm{mL}$ of carrier DNA.

147 Approximately 6,000 Ura+ yeast transformants were collected for pooled competition 148 experiments and for PacBio sequencing (Figure 1A).

Linking barcodes with full-length variants

Plasmids were extracted from the yeast transformant pool using Zymoprep Yeast

152 Plasmid Miniprep II (Zymo Research). Plasmid fragments containing the barcode and variant 153 were isolated using M13/pUC primers with KAPA HiFi Hotstart Readymix and the following 154 cycling conditions: $95^{\circ} \mathrm{C}$ for $3 \mathrm{~min}$, then 13 cycles of $98^{\circ} \mathrm{C}$ for 20 seconds, $60^{\circ} \mathrm{C}$ for 15 seconds, 155 and $72^{\circ} \mathrm{C}$ for 4 minutes. The final product was extracted from a $0.5 \%$ agar gel using Qiagen's 
Gel Extraction kit and cleaned using Ampure PB beads (Pacific Biosciences). Two PacBio libraries were made using the SMRTbell ${ }^{\mathrm{TM}}$ Template Prep Kit 1.0 (Pacific Biosciences) and sent to University of Washington PacBio Sequencing Services for sequencing and Sequel II circular consensus sequence (CCS) analysis.

BAM files of CCS reads were aligned to the plasmid reference file using BWA/0.7.13 mem (Li, 2013). Reads that were aligned to the reference were piped to a new BAM file with Samtools/1.9 (Li et al., 2009). These reads were also analyzed with cigar strings to validate alignment of PacBio reads. From there, the barcodes were extracted, and a barcode-variant map was generated that contained a file with all of the barcode-variant reads and all of the highest quality reads for each barcode, as previously described (Matreyek et al., 2018). Since the resulting barcode-variant map still showed a considerable number of insertion and deletion errors, we used a multiple sequence alignment of all the reads that shared the same barcodes to eliminate additional sequencing errors. Alignments were done using MUSCLE (v.3.8.31) (Edgar, 2004). Any further ambiguous nucleotides were resolved by performing a pairwise alignment against the highest quality read (EMBOSS Needle v. 6.4.0) (Needleman and Wunsch, 171 1970).

To match PacBio reads to strains in the 1,011 collection, reference sequences were first

173 extracted from the GVCF in the 1,011 collection genome data using BCFtools consensus. We

174 then used regular expressions to search for reads that were putatively derived from these

175 reference sequences. We removed barcodes that contained only one CCS read or were not

176 represented in our barcode sequencing analysis (Figure 1B).

\section{Pooled library competition in chemostats}

Sulfate-limited media (3mg/L ammonium sulfate) was prepared as previously described

180 (Gresham et al., 2008; Payen et al., 2014). Four $50 \mathrm{~mL}$ chemostat culture vessels were filled 181 with $20 \mathrm{~mL}$ of media at $30^{\circ} \mathrm{C}$ and inoculated with $1 \mathrm{~mL}$ of the yeast transformant pool. This 
culture was grown for 24 hours, after which the pumps were turned on and the culture switched to a continuous culture system at a dilution rate of about 0.17 volumes per hour $(\sim 3.4 \mathrm{~mL} / \mathrm{hour}$

184 in a $20 \mathrm{~mL}$ culture). Samples were taken twice a day for 5 days, or about 25 generations. For each sample, $1 \mathrm{~mL}$ was stored in $25 \%$ glycerol at $-80^{\circ} \mathrm{C}$, and another $1 \mathrm{~mL}$ was used for plasmid extraction (Figure 1C).

\section{Barcode sequencing and analysis}

For each time point and replicate from the pooled library competition, plasmids were again extracted using the Zymoprep Yeast Plasmid Miniprep kit. One replicate was discarded due to technical errors. Barcodes were isolated and amplified using forward oligo 25 and indexed reverse oligos 26-40 and 120-128 that included Illumina Nextera sequencing adaptors. KAPA HiFi Hotstart Readymix was used with $1 \mu \mathrm{L}$ of $1 \mathrm{X}$ SYBR ${ }^{\mathrm{TM}}$ Green I and the following PCR cycles: $95^{\circ} \mathrm{C}$ for $3 \mathrm{~min}$, then $17-19$ cycles of $98^{\circ} \mathrm{C}$ for 20 seconds, $60^{\circ} \mathrm{C}$ for 15 seconds, and $72^{\circ} \mathrm{C}$ for 15 seconds. The reaction was run on a Bio-Rad MiniOpticon (Bio-Rad) to avoid overamplification. PCR products were cleaned using Ampure XP Beads (Agencourt) and quantified using the KAPA Library Quantification Kit for Illumina ${ }^{\circledR}$ Platforms (Roche). Libraries were sequenced on a NextSeq sequencer (Illumina) with sequencing oligos 41 (Read 1), 44 (Read 2), and 100 (Index). Paired-end reads were merged using PEAR/0.9.5 (Zhang et al., 2014). Using FitSeq, we calculated the fitness of each barcode for each given replicate (Li et al.,

201 2018). FitSeq normalizes each pool to account for experimental error between replicates,

202 providing a more accurate readout of fitness. The fitness values were then normalized by the 203 average fitness of barcodes associated with the wild-type (S288C) alleles. The effects of single 204 mutations were also compared with predicted consequences of mutations from mutfunc (Wagih 205 et al., 2018) (Figure 1C). 
To assess fitness of strains carrying individual alleles, $300 \mu \mathrm{L}$ of a liquid culture of each strain was inoculated into a chemostat containing $20 \mathrm{~mL}$ of sulfate-limited media at $30^{\circ} \mathrm{C}$. For

210 each of the competitors, one competitor strain contained a plasmid with an extra copy of SUL1

211 and the other isogenic strain contained a neutral GFP marker. Additionally, competition

212 experiments of strains carrying each allele being assayed were conducted in at least two

213 biological replicates. Cultures were grown for 24 hours before switching to a continuous culture

214 system. Once cultures achieved steady state, the competing cultures were mixed at a 1:1 ratio.

215 Cultures were competed for 15 generations after mixing and sampled twice daily (approximately

216 every 3-6 generations). For each sample, cultures were assayed for percent GFP cells with a

217 BD Accuri C6 flow cytometer (BD Biosciences). Competitive fitness values were calculated by

218 plotting In(number of dark cells/number of GFP+ cells) over about 25 generations and taking the

219 linear slope of the linear regression from this data.

224 sulfate abundant media was prepared by adding ammonium sulfate (to $5 \mathrm{~g} / \mathrm{L}$ ) to the sulfate-

225 limited media. To ensure the depletion of sulfate in the cells, all 1,011 natural isolates were

226 grown overnight ( $\sim 14$ hours) on solid sulfate-limited media. The isolates were then replicated in

227 quadruplicate on solid sulfate-limited and sulfate-abundant media. Photos of the colonies were

228 taken every 12 hours for 3 days and the R package gitter was used to calculate the size of each

229 colony in the photos (Wagih and Parts, 2014). For each time point on both limited and abundant

230 conditions, we subtracted the colony size at the first time point from the colony size at

231 subsequent time points (colony size $=$ size $_{t}-$ size $_{t=0}$ ). Growth rates were calculated by taking the

232 average of the ratio of the colony size in limited media over the colony size in abundant media

233 across 72 hours. 
Phylogenetic tree generation and sequence analysis

To generate our phylogenetic trees, SUL1 sequences from the 1,011 strains and from S. paradoxus strain CBS432 were aligned using MUSCLE (Edgar, 2004). The genetic distances

238 for SUL1 alleles were calculated using the maximum-likelihood-based distances through

239 DNADIST in the PHYLIP package (Felsenstein, 2005). A gene tree for SUL1 was then

240 generated using the NEIGHBOR program, and the final tree was visualized and annotated using

241 R/ggtree (Yu et al., 2017).

242 To determine the prevalence of loss-of-function mutations across all 1,011 strains, we

243 used sequences from the core ORFs in the pangenome as reference sequences and identified

244 which strains were homozygous for premature stop codons in each of the core ORFs (Peter et

245 al., 2018). Premature stop codons that occurred in the last $90 \%$ of an ORF were not included,

246 as previous studies have shown that these mutations would not necessarily cause a significant

247 loss of function (Bergström et al., 2014). Gene Ontology (GO) analysis was conducted using

248 Yeastmine (accessed May 22, 2020) and both Benjamini-Hochberg and Bonferroni test

249 corrections were used to account for multiple testing (Balakrishnan et al., 2012).

Data availability

252 Raw sequencing data can be found in the Sequencing Read Archive (BioProject Accession

253 PRJNA681436 https://www.ncbi.nlm.nih.gov/bioproject/PRJNA681436). Scripts and

254 Supplemental Tables used for this paper can be found at

255 https://github.com/dunhamlab/SUL1 natural variants. All alleles, matched strains, barcodes,

256 fitness, coding mutations, and noncoding mutations can be found in Supplementary Table 3.

\section{Results}

259 Allele library curation and characterization 
When sulfate is a limiting nutrient, S. cerevisiae increases expression of SUL1, which encodes a high-affinity sulfate permease that increases the intake of sulfate molecules into the cell. Previously, we measured the competitive fitness of SUL1 alleles isolated from 10 different wild yeast isolates (Payen et al., in preparation). We found that these alleles confer a wide range of fitness: some had loss-of-function phenotypes while others performed better than the allele found in the reference strain S288C. In order to determine if this wide variation was representative across the entire species and whether it correlated with features such as the

267 environment from which each strain was isolated, we set out to survey SUL1 functionality 268 across a bigger sample of natural isolates. For this study, we used the 1,011 S. cerevisiae strain collection, which was curated from a variety of geographical and ecological origins (Peter et al., 2018). In addition, the collection contains at least 250 unique alleles of SUL1 with 354 variable

271 sites in the gene. Alleles contain 11 polymorphisms on average vs. the reference allele, with the

272 most polymorphic allele having 79 mutations. Therefore, these factors make SUL1 a powerful

273 tool for us to better understand the natural variation of a single gene in S. cerevisiae

274 populations.

In our previous studies, the fitness of individual SUL1 alleles was measured by

276 transforming the reference strain with an additional copy of a SUL1 allele on a low-copy

277 plasmid, and the resulting strain was competed against an isogenic GFP-marked strain under

278 sulfate limitation (Payen et al., in preparation) (Sanchez et al., 2017). While this assay is reliable

279 and consistent, it would be difficult and unrealistic to scale to measure hundreds of alleles.

280 Thus, we developed a high-throughput, multiplexed approach that allows us to simultaneously

281 measure these fitness values directly (Figure 1). To do this, we pooled the 1,011 isolates

282 together, extracted genomic DNA, and used barcoded primers binding to conserved regions to

283 isolate and amplify all natural alleles of the SUL1 gene. These sequences were cloned en

284 masse onto low-copy CEN/ARS plasmids to create an allele library and used to transform the

285 reference strain (FY). The resulting library contained approximately 6,000 barcodes for an 
estimated 250 unique alleles (24X coverage) to ensure complete coverage and internal replicates (Figure 1A).

We used PacBio long-read circular consensus sequencing (CCS) to pair barcodes with their respective alleles (Figure 1B). Although PacBio CCS has drastically improved and decreased sequencing errors over the past few years, we found that many reads still contained errors that were especially noticeable in the form of insertions and deletions. To further eliminate these sequencing artifacts, we performed multiple sequencing alignments on CCS

293 reads that shared the same barcode, and used those to derive new consensus sequences. In

294 total, our analysis produced 8,386 barcode-variant pairs, which we determined was still an

295 overestimate given our library size of $\sim 6,000$ barcodes. We removed consensus reads that only 296 appeared once to eliminate false positives or negatives in our downstream analysis, with 3,787 barcodes remaining.

Among these 3,787 barcodes, we identified 407 unique alleles in our library. Of these variants, we were able to match 228 alleles to at least one strain in the 1,011 strain collection,

300 with a total of 880 strains that had at least one matched allele in the library (Supplemental

301 Figure 1). To determine how well this library reflected the polymorphisms in the strain 302 collection, we plotted the correlation of polymorphism frequency in both the variant reference 303 sequences and library sequences and found that these values were highly correlated 304 (Pearson's correlation, r=0.978, Supplemental Figure 2). Correlation values were similar for 305 polymorphisms in all regions of the gene: the 5'-UTR, coding region, and 3'-UTR were all well306 correlated (Pearson's correlation, $r=0.956,0.980$, and 0.993, respectively). Of the 354 variable 307 sites found in the reference sequences, only 45 of them were not detected in the allele library, 308 nine of which were rare polymorphisms. Our pipeline did not reveal any de novo mutations that 309 could have resulted from PCR or sequencing artifacts. 


\section{A. Generate allele library}

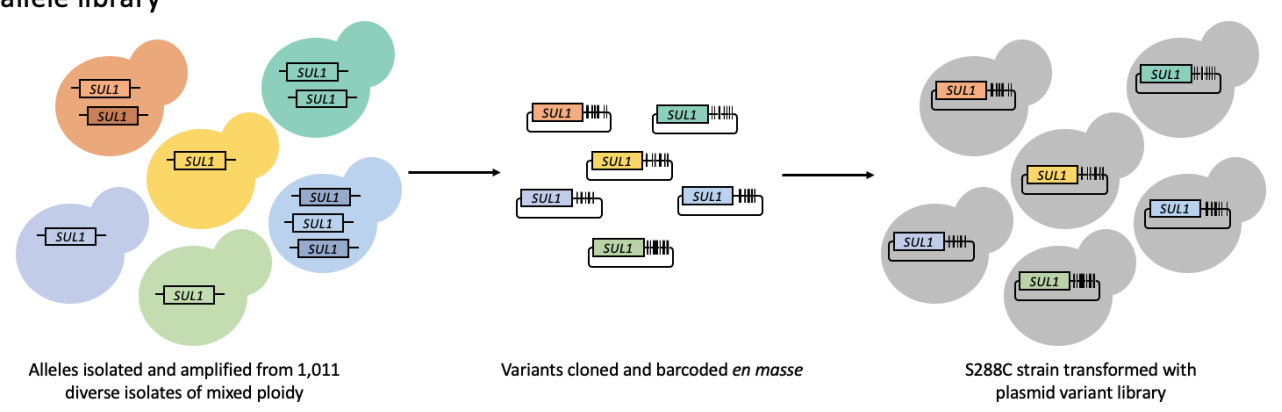

B. Link barcodes to variants
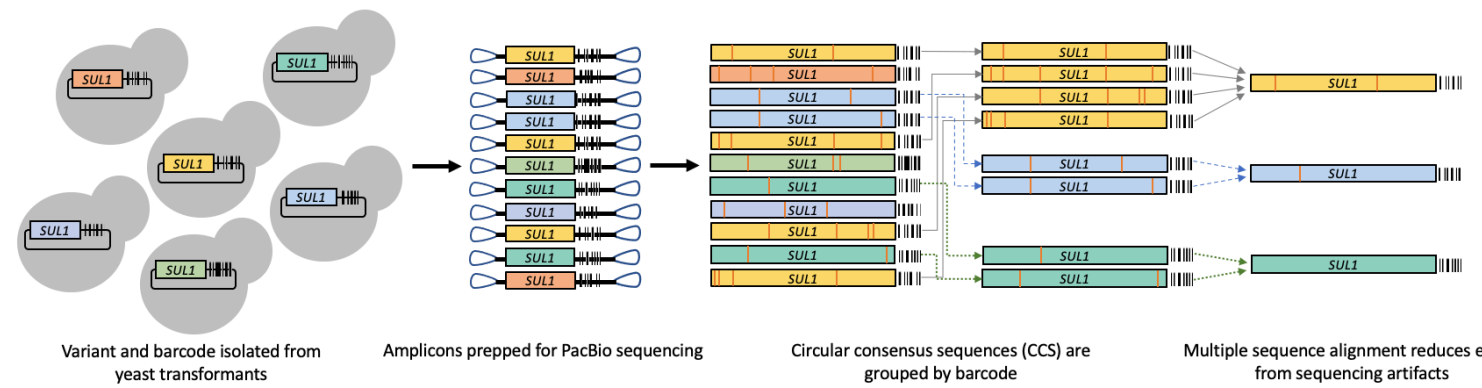

yeast transformants
yount and isoled from

Circular consensus sequences (CCS) are grouped by barcode

Multiple sequence alignment reduces errors from sequencing artifacts
fromce alignment reduces

\section{Measure allele fitness}

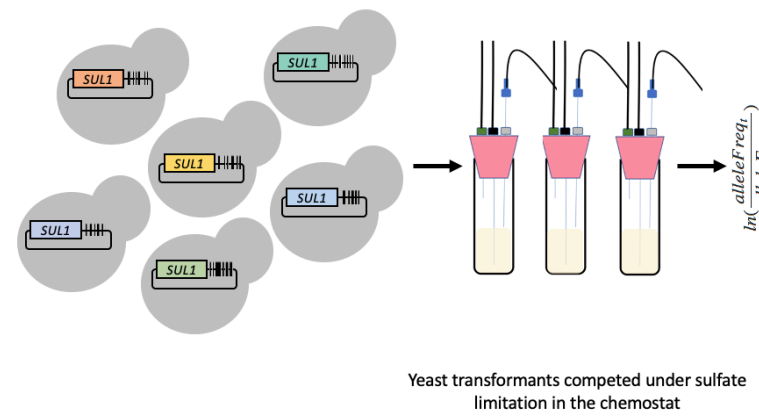

limitation in the chemostat

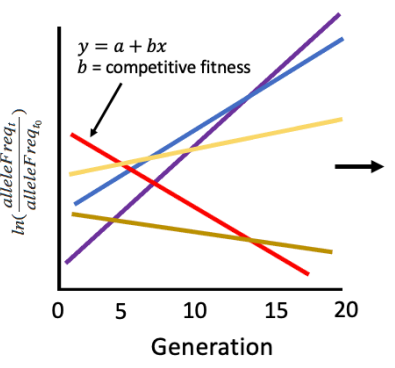

Changes in allele frequencies used to infer fitness. FitSeq corrects for poolspecific biases.

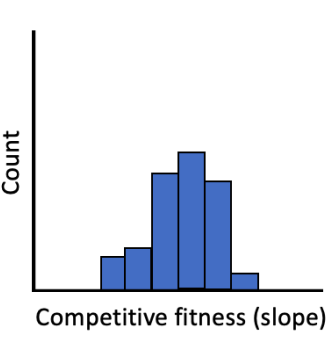

Fitness of each allele used to generate species-wide fitness distribution

Figure 1. Workflow for assaying natural variants in the 1,011 strain collection. A) The S288C lab strain is transformed with SUL1 natural allele barcoded plasmid library. B) PacBio long-read sequencing is used to link barcodes with variants. C) Transformants are competed together under sulfate limitation. Barcode sequencing every 3-4 generations is used to calculate the abundance of each variant and its respective competitive fitness.

We were also unable to capture the alleles from 23 strains that were identified to have

311 SUL1 introgressed from Saccharomyces paradoxus. This was likely due to these sequences

312 being more highly diverged and therefore unable to hybridize with the primers that were

313 designed. However, for completeness, we were still able to measure the functionality of the

314 introgressed SUL1 alleles using our lower throughput method of direct competitions, as

315 described below. 
To determine the fitness landscape of all the SUL1 alleles present in our allele library,

318 we competed the library of yeast transformants in a continuous culture system under sulfate-

319 limited media. Samples from 12 timepoints across four replicates were collected every 3-4

320 generations. For each sample, we extracted the plasmids from sampled cultures and

321 sequenced the ba rcodes using Illumina short-read sequencing. By tracking the change in

322 barcode frequencies over the 12 timepoints, we determined the competitive fitness values for

323 strains carrying each allele (Figure 1C). The calculated competitive fitness of the three

324 replicates showed strong correlation and reproducibility (Supplemental Figure 3).

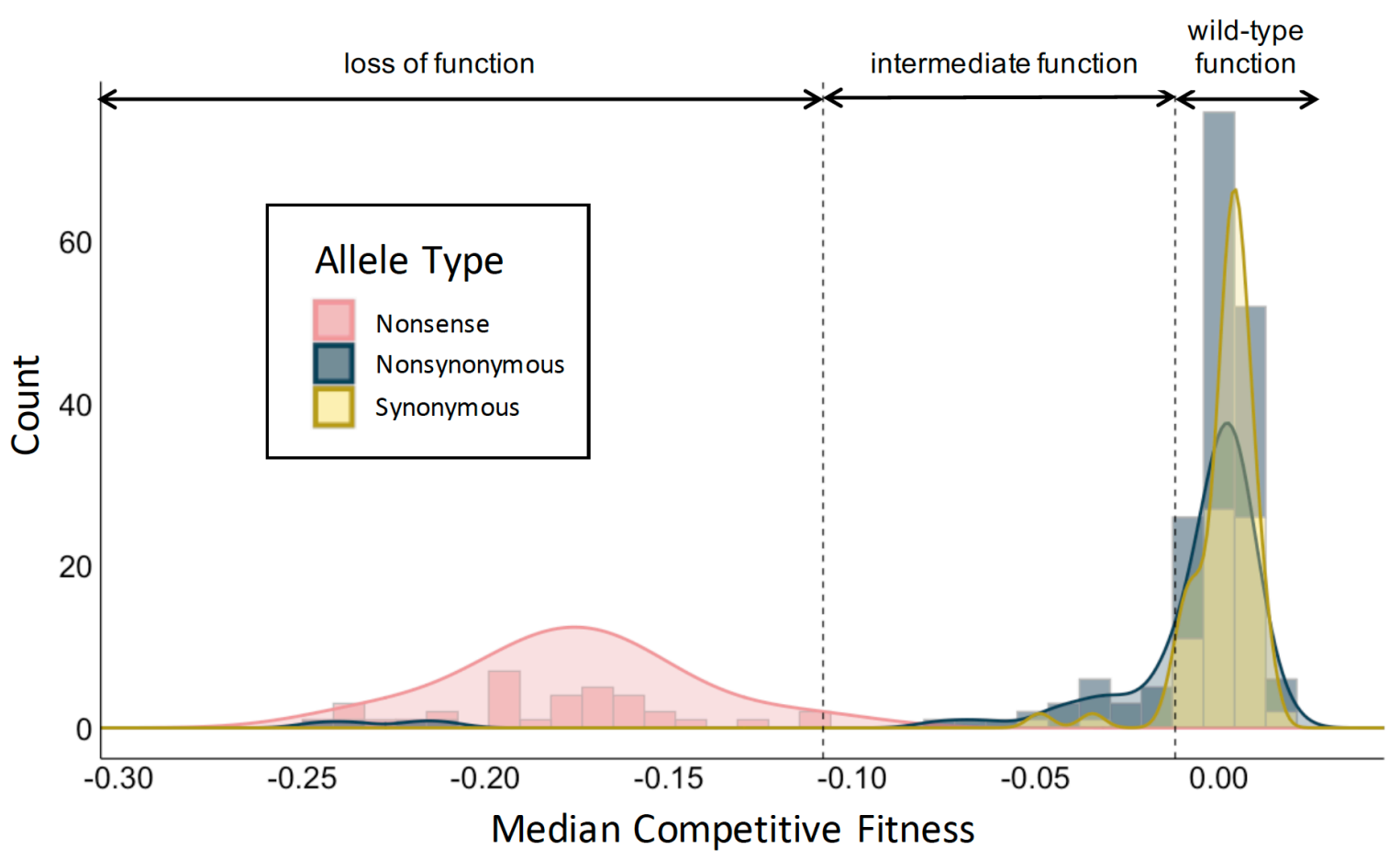

Figure 2. Species-level distribution of fitness effects of natural SUL1 alleles. Lab strain S288C yeast transformed with an allele library of SUL1 cloned onto a low-copy plasmid were competed in sulfate-limited media in the chemostat. The log-fold change in proportions of each barcode across 12 timepoints were measured through barcode sequencing and used to calculate competitive fitness. Alleles categorized as nonsense alleles may also contain synonymous and nonsynonymous polymorphisms. Those grouped as nonsynonymous alleles may contain synonymous polymorphisms, but do not have premature stop codons. Synonymous alleles do not have nonsynonymous or nonsense polymorphisms. All alleles may contain polymorphisms in the promoter or 3'UTR. Loss-of-function alleles were defined as having a fitness lower than the highest-fit allele with a premature stop codon. Wild-type function alleles have a fitness higher than the lowest-fit synonymous allele. 
In our barcoded library, 863 of 3,787 barcodes were associated with alleles identical to that of the S288C reference strain. We normalized all fitness values to the average fitness of these wild-type alleles $(0.0097$, standard deviation 0.0698). Reassuringly, we found that many barcodes with lower fitness values (fitness < -0.03) were largely associated with alleles containing natural premature stop codons (Figure 2). In fact, upon analyzing the sequences in each strain, we found 74 strains that are homozygous for premature stop codons in their SUL1 alleles. Among the 31 alleles with premature stop codons, fifteen occur in amino acid positions

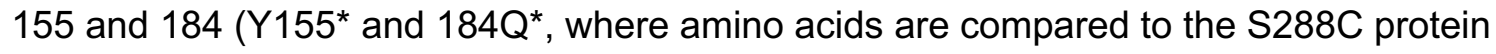
333 sequence).

Due to the wide range in fitness of alleles with premature stop codons, we investigated whether stop codons that occurred earlier in SUL1 have a greater impact on function. We found that the location of stop codons in SUL1 did not dictate the deleterious effects of containing a nonsense mutation (Supplemental Figure 4a). However, the fitness of alleles with premature stop codons at amino acid position 671 consistently have much lower fitness compared to others with premature stop codons elsewhere. This stop codon occurs in the predicted extracellular STAS (sulfate transporter and anti-sigma factor antagonist) domain, which is

341 thought to be crucial for metabolism sensing, and may be further impacting sulfate transport 342 under sulfate limiting conditions (Sharma et al., 2011). We compared the standard deviations among barcodes that shared the same loss-of-

344 function alleles to that of barcodes that shared the same wild-type alleles (Supplemental

345 Figure 4b). The barcodes linked to loss-of-function alleles do vary more in fitness (Welch two 346 sample t-test, $p<0.005)$, although we attribute this variance to increased errors that occur when 347 measuring fitness on a log scale. In regard to magnitude, the barcode counts are reliable, but 348 the barcode counts tend to be less accurate when frequencies are low and continue to decrease 349 through later time points. 


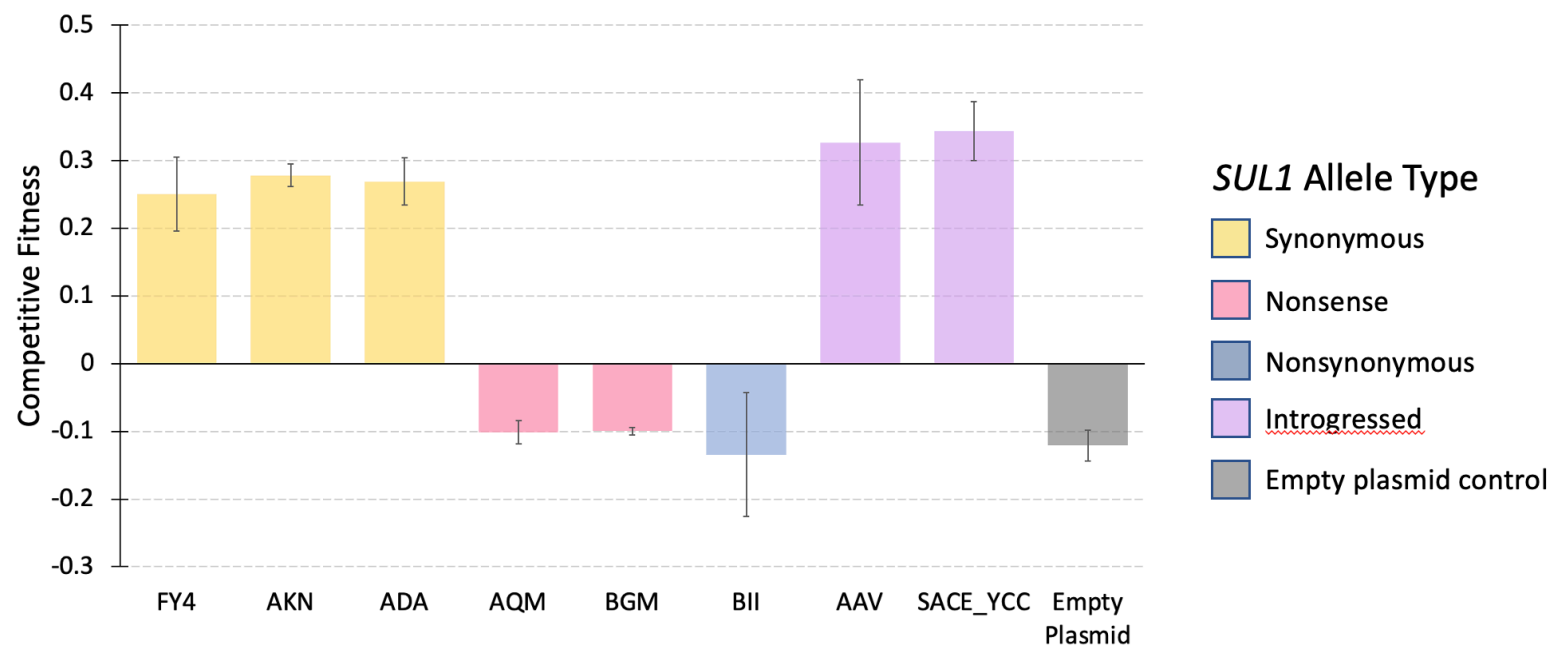

Strains from which SUL1 alleles were derived

Figure 3. Validation of pooled competition through direct competitions of selected natural SUL1 alleles. S288C strains transformed with a specific SUL1 allele on a low-copy plasmid were individually competed against an isogenic GFP-marked strain in the chemostat. Fitness values were calculated by tracking the log-fold change in proportion of non-fluorescent strains and fluorescent strains over 20 generations. These values were used to validate select alleles and their phenotypes observed in the pooled competition. Alleles were selected based on definitive categorization in wild-type-like (pooled competitive fitness close to 0 ) or loss-of-function (pooled competitive fitness less than -0.10) phenotypes. Of the loss-of-function alleles, AQM and BGM have premature stop codons while BII is loss-of-function due to nonsynonymous polymorphisms. SUL1 alleles in AKN, ADA, and BII were done in a prior experiment (Payen et al., in preparation).

In addition to stratifying alleles with premature stop codons and alleles with wild-type phenotypes, we identified alleles with nonsynonymous polymorphisms that also result in a loss of function. For instance, alleles that have a single polymorphism resulting in a T669K amino

353 acid substitution show a loss of function. We also found that alleles with A454P and D483N and

354 alleles with S699L substitutions (and no additional nonsense or promoter polymorphisms) have

355 a loss of function phenotype in our pooled library. Alleles with their polymorphism information,

356 corresponding strain information, and measured fitness values can be found in Supplementary

\section{$357 \quad$ Table 3.}

We assessed how well the fitness values are reflected in direct competitions by selecting SUL1 alleles from seven isolates and cloning them individually on the same low-copy plasmid. We transformed S288C haploid yeast with these individual plasmids and competed each allele 
selected to validate a wild-type-like phenotype and corresponded to the values calculated in the pooled competition. Three other alleles selected showed a loss-of-function phenotype in the pooled competition, which was reflected in the direct competitions. Two of these alleles contained a deletion that resulted in a frameshift (from strains BGM and AQM), and the third allele had nonsynonymous mutations (from strain BII). The BII strain has previously been evolved through sulfate limitation for 150 generations, and it was found that a natural polymorphism that results in a P296L change is responsible for the loss-of-function phenotype (Payen et al., in preparation). In each case, we found the results of the direct competitions recapitulated those found in our pooled competition. used the same approach of a direct competition to assay introgressed allele functionality. After validating the fitness of the SUL1 orthologue from S. paradoxus in the S. cerevisiae background, which has previously shown high fitness (Sanchez et al., 2017), we also tested the

375 fitness of two alleles that show signatures of introgression from S. paradoxus. The two

376 introgressed alleles, despite having over 40 amino acid differences compared to the reference

377 allele, also have a wild-type phenotype (Figure 3).

The fitness distribution across the natural alleles shows alleles with only synonymous site changes in the coding region that nevertheless have a lower competitive fitness compared

382 to strains carrying the wild-type coding sequence from the reference strain (Figure 2). We

383 reasoned that these alleles may instead carry functional differences in the noncoding

384 sequences. We found that these alleles share the n.-456G>A polymorphism, and upon further

385 inspection discovered that this SNP is only present in alleles (including those with additional

386 nonsynonymous SNPs) with lower competitive fitness values under sulfate limitation (median

387 competitive fitness $=-0.04)$. Since this competitive fitness value is not as low as alleles with 
premature stop codons (median competitive fitness $=-0.17$ ), it is indicative of an intermediate phenotype. This SNP occurs in a putative Cbf1-binding motif, and binding of this Cbf1 transcription factor has been shown to be important for growth in sulfate limiting conditions (Rich et al., 2016; Siggers et al., 2011). The SNP also decreased fitness in a SUL1 promoter mutagenesis study, further supporting the functional effects of changes in this motif (Rich et al., 393 2016).

We used the highest fitness of an allele that contains a premature codon (median competitive fitness $=-0.108$ ) and the lowest fitness of alleles without promoter or nonsynonymous polymorphisms (median competitive fitness $=-0.0120$ ) to establish a range for other alleles with intermediate phenotypes. Twenty-two unique alleles show an intermediate phenotype, and 9/20 alleles with nonsynonymous polymorphisms also have the n.-456G>A polymorphism. Using these benchmarks, we also identify nonsynonymous changes that do not confer a complete loss of function.

The observation of promoter mutations affecting phenotype in sulfate limitation led us to

402 inspect how much promoter polymorphisms in general contribute to the fitness values observed

403 across the entire allele library. We compared the standard deviation in fitness for sequences

404 that share the same coding sequence to the standard deviation in fitness for sequences that 405 share the same promoter sequences. We found that the coding sequences seemed to more 406 consistently determine fitness of a strain under sulfate limitation (Figure 4a). That is, alleles with 407 the same promoter sequences had a greater variance in fitness values. Furthermore, alleles 408 that shared the same coding sequences but differed in promoter sequences showed few 409 significant differences in fitness (Figure 4b). Finally, despite the fact that the promoter 410 mutagenesis study found mutations that could improve fitness under sulfate limitation, we did 411 not identify such polymorphisms among our natural variants. 
A

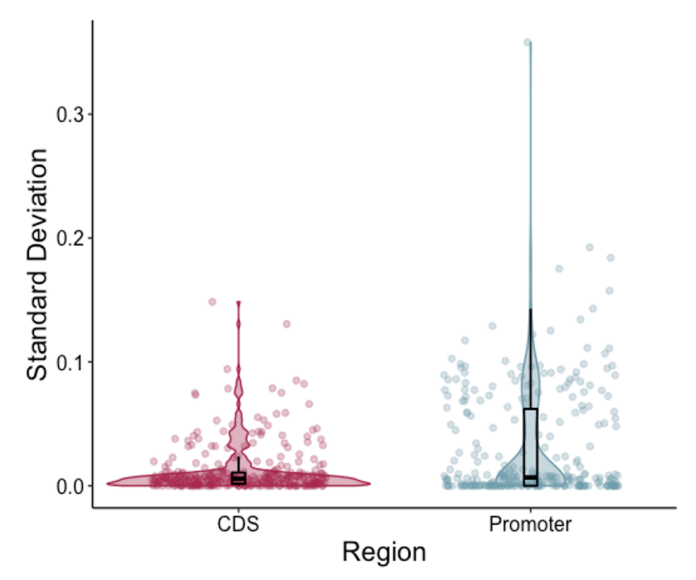

B

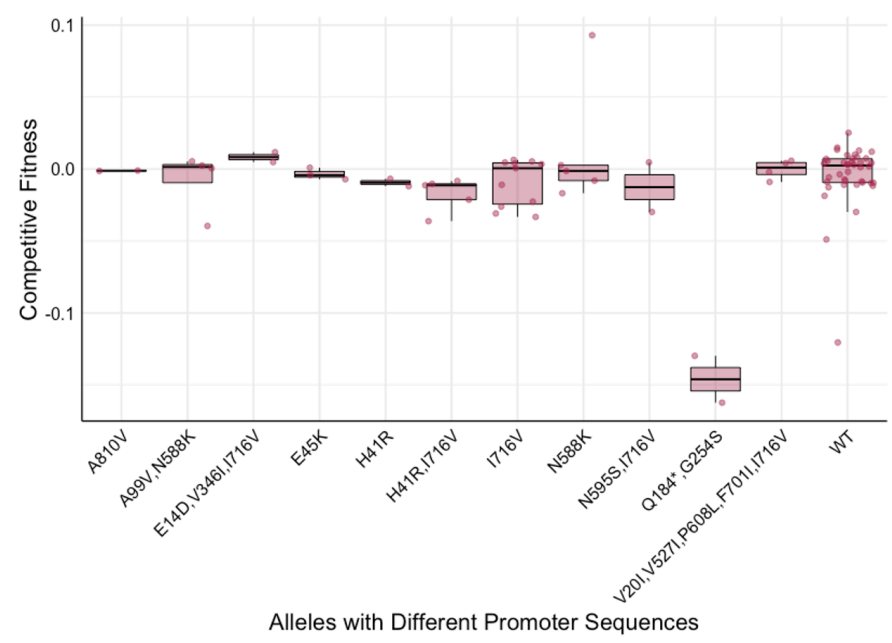

Figure 4. Coding polymorphisms are more useful for predicting deleterious effects compared to those in the promoter of SUL1. A) Violin plots of the standard deviations of the competitive fitness for barcodes grouped by those that share the same coding sequence compared with the standard deviation of those that share the same promoter sequence. B) Boxplots of competitive fitness of the sequences that share the same coding sequence but differ in the promoter sequences.

Comparing competitive fitness with mutfunc

With nonsense mutations, loss of function can be predicted based on sequence alone.

414 However, predicting the functional effects of other mutations based on sequence alone is much

415 more challenging. To determine how well these fitness values were reflected in functional

416 computational predictors, we used mutfunc to compare our results to predicted functional

417 effects. For each variant, we took the most putatively detrimental mutation and compared its

418 value to the fitness values calculated in our pooled competition assay. While the SIFT scores

419 and our fitness values themselves showed very little correlation (Supplementary Figure 5a),

420 we found that most alleles with a loss-of-function phenotype had a low SIFT score

421 (Supplementary Figure 5b). Interestingly, many mutations that SIFT predicted would be

422 detrimental actually had a wild-type-like phenotype under sulfate limitation. This highlights the

423 value of experimentally measuring the function of variants, especially in cases where we need

424 to consider the functional impacts of multiple polymorphisms on the same haplotype. 


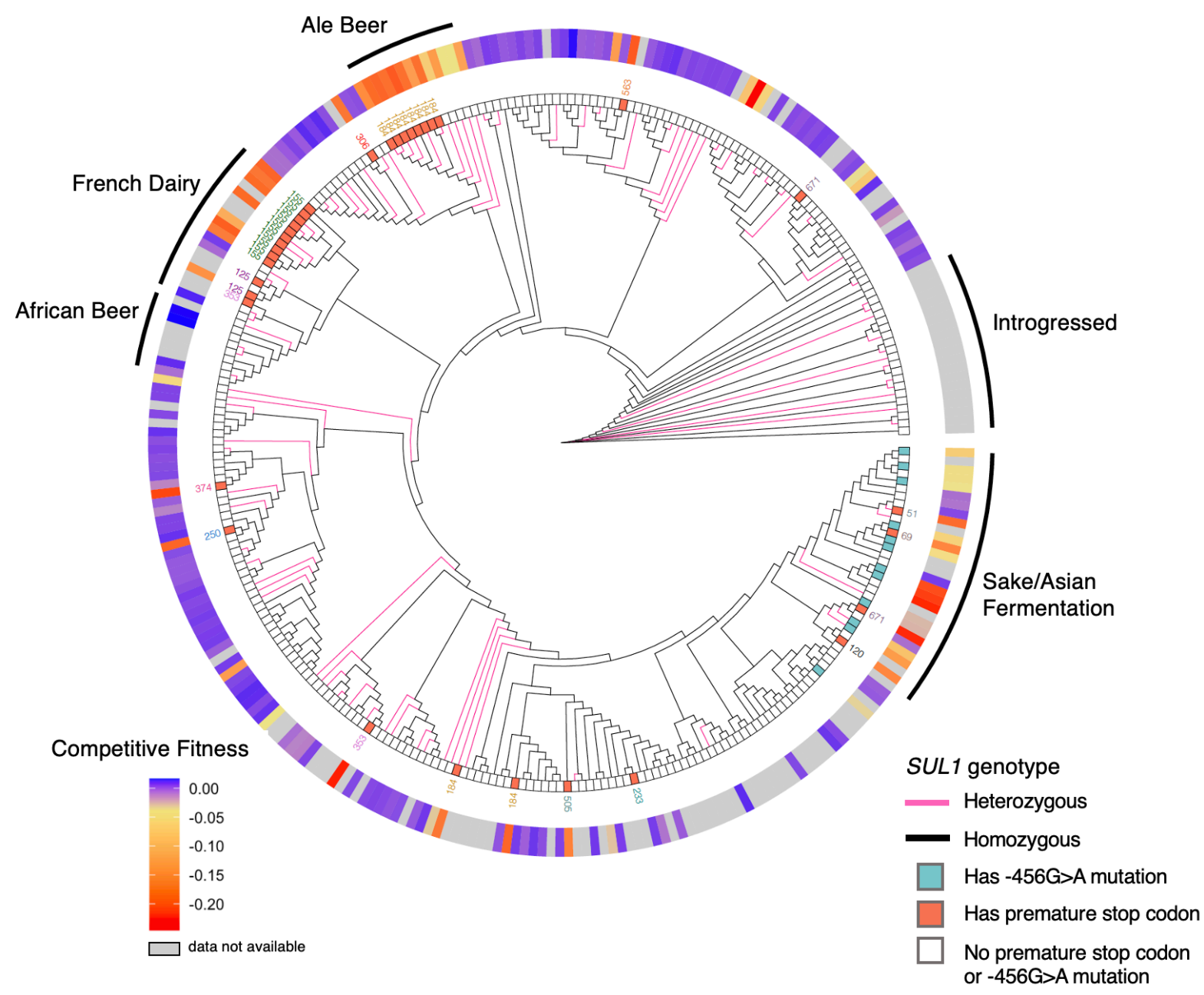

Figure 5. Neighbor-joining gene cladogram generated through PHYLIP using unique genotypes of SUL1 in the 1,011 strain collection. French dairy and sake/Asian fermentation clades both show multiple independent instances of loss-of-function mutations. A stop codon at amino acid position 184 occurs independently in different strains. Color of edges (pink or black) indicates whether genotype for those terminal nodes are homozygous or heterozygous. Heterozygous alleles can be derived from diploid, triploid, tetraploid, or even pentaploid strains. Boxes directly adjacent to terminal nodes indicate the genotypes that are homozygous for a premature stop codon (red) or a -456G>A mutation (cyan). Flanking boxes of genotypes with premature stop codons are numbers indicating where in the amino acid sequence the premature stop codon occurred. The ring surrounding the tree denotes the mean SUL1 competitive fitness values for a given strain's allele on a purple (wild-type-like fitness) to red (loss-of-function fitness) gradient. Labeled regions are generalizations for what comprises most of those clades.

Phylogenetics and sequence analysis of natural SUL1 alleles

To assess phenotypic patterns of SUL1 on the population level, we annotated a

427 distance-based gene tree of SUL1 (Figure 5) with the competitive fitness values we calculated

428 from our pooled competition assay. In our gene tree, we used the SUL1 allele of

429 Saccharomyces paradoxus (CBS432) as the outgroup. We removed branch lengths from these 
430 trees to simplify interpretations. Using these annotated trees, we are able to interpret phenotype

431 in relation to ecological origins and phylogenetic relationships (Figure 5). We firstly looked at

432 the strains homozygous for premature stop codons in SUL1. The polymorphism that results in

433 Q184* does not occur in a singular clade, reducing the possibility that this premature stop codon

434 arose in prevalence as a result of drift or identity by descent. Alleles with $\mathrm{Y} 155^{\star}$ are primarily

435 present in strains isolated from dairy environments in Normandy, France; however, not all dairy

436 strains share the same nonsense mutation (Figure 6). Two other strains derived from dairy,

437 AQM and BGM, instead have the L125* frameshift mutation. This pattern suggests that a loss-

438 of-function mutation could be beneficial in a dairy environment.

439 The majority of strains with the detrimental promoter mutation $\mathrm{n} .-456 \mathrm{G}>\mathrm{A}$ were isolated

440 from sake or Asian fermentation strains. Additionally, many strains in this clade have a

441 premature stop codon and or nonsynonymous polymorphisms that result in loss of function,

442 which would again support the idea that there may be a trade-off for having a loss-of-function

443 SUL1 allele since more than one loss-of-function allele sequence exist among these strains.

444 Based on the distribution of deleterious alleles over the phylogeny, we wondered if these

445 allele differences would lead to phenotype differences when the alleles were in their native

446 strain context. We grew all isolates (unmodified) from the 1,011 strain collection on solid

447 minimal media agar plates under sulfate limitation and compared the growth rates to that of the

448 strains pinned on sulfate-abundant minimal media. Interestingly, we found little to no correlation

449 between the growth rates of strains and the competitive fitness values of their SUL1 alleles

450 (Supplemental Figure 6a,b). We additionally looked for growth patterns among ploidy,

451 geographical origins, and clade and found no patterns related to these groupings

452 (Supplemental Figure 6c). These results argue that additional background effects beyond the

453 SUL1 locus matter for determining fitness in sulfate limitation. Measuring the fitnesses of the

454 allele library in additional strain backgrounds may help further characterize this genetic

455 complexity. 
457 the value was low $(d N / d S<0.2)$, suggesting that there may be purifying selection on SUL1.

458 Additionally, Tajima's $D$ statistic suggests that SUL1 is unlikely to be evolving neutrally $(D=$ -

459 2.85). This may indicate that this locus has not reached equilibrium after a bottleneck in the past

460 and is still undergoing expansion. The neutrality index calculated from the McDonald-Kreitman

461 test indicated no evidence of selection $(\mathrm{NI}=1.117$, Fisher's exact two-tailed test, $\mathrm{p}=0.625)$;

462 however, there are mutations in the S. cerevisiae population that are slightly and fully

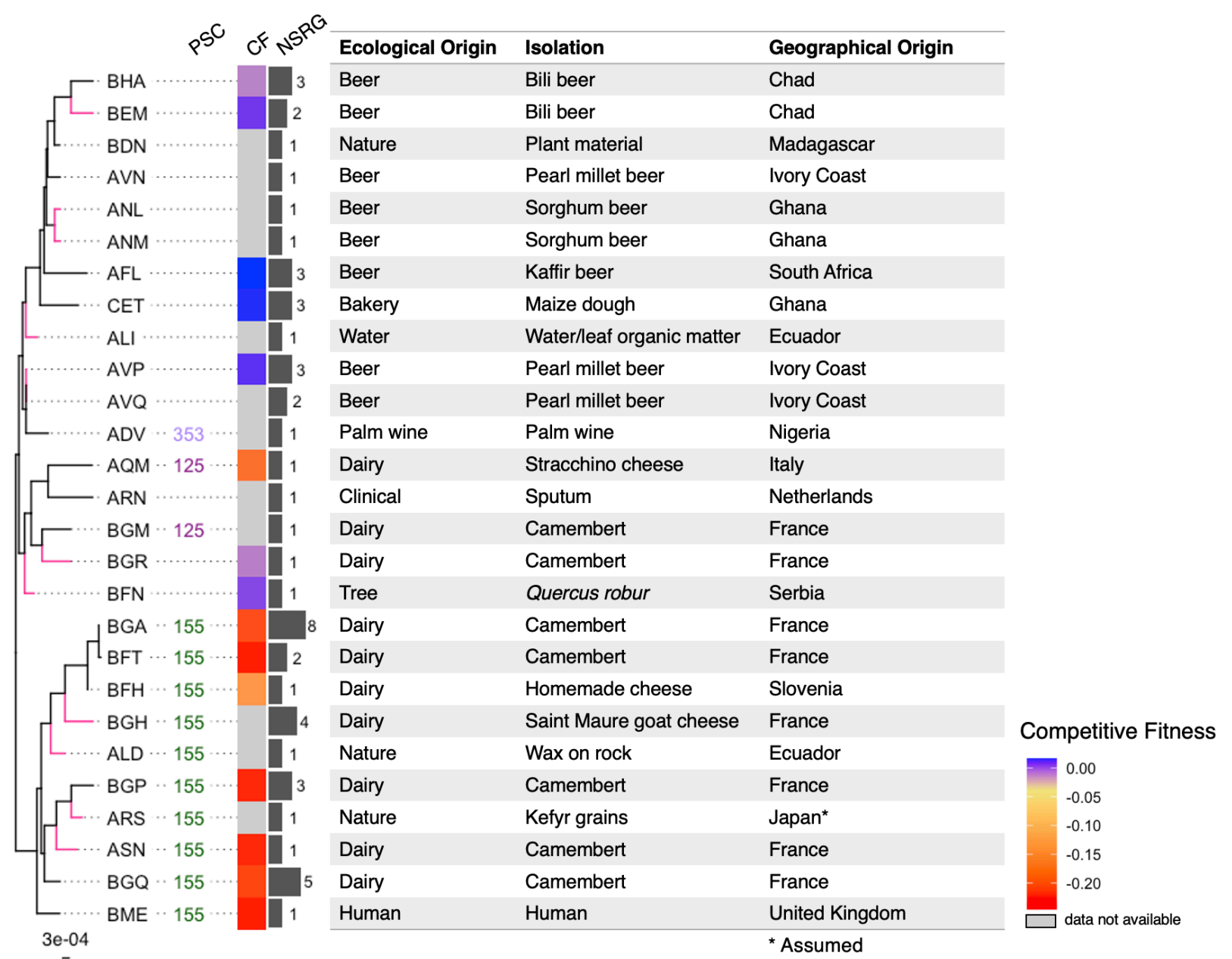

Figure 6. Dairy and African beer subtree of the 1,011 SUL1 genotypes. Although dairy strains AQM and BGM share a more recent common ancestor to African beer strains, they show different but independent and homozygous loss-of-function polymorphisms. Color of edges (pink or black) indicate whether genotype for those terminal nodes are homozygous or heterozygous. PSC, amino acid site with premature stop codon (homozygous); CF, competitive fitness; NSRG, number of strains represented by genotype. Boxes around terminal nodes indicate the genotypes that are homozygous for a premature stop codon (red) or a $-456 \mathrm{G}>\mathrm{A}$ mutation (cyan). Scale (bottom left) indicates number of nucleotide substitutions per site. 
deleterious, which have been shown to cause errors in predictions of adaptive evolution using this test (Charlesworth and Eyre-Walker, 2008). mutations, we determined the frequency of likely deleterious premature stop codons at all loci in

467 the 1,011 strain collection sequences. Using the sequencing data curated in the 1,011 S.

468 cerevisiae strains, we analyzed the coding sequences of genes in the pangenome for premature 469 stop codons that occurred in the first $90 \%$ of the gene. We excluded genes that either did not 470 appear in the pan-genome or contained premature stop codons in the pan-genome reference

471 sequences. Grouping these genes enriched in premature stop codons by ecological origins, we

472 found that dairy strains tended to have a consistently higher number of genes that are

473 homozygous for premature stop codons compared to strains isolated from other ecological

474 origins (Supplemental Figure 7). This is consistent with previous studies that identified

475 enriched loss-of-function alleles among dairy strains that were a result of drift and are important

476 for trait variation (Legras et al., 2018; Zorgo et al., 2012). Of all the genes in the pangenome,

4772,465 genes contain a premature stop codon in at least two strains, with 862 of these genes

478 containing premature stop codons in more than 20 strains. Gene Ontology (GO) term analysis

479 revealed that 158 of these 862 genes are involved in ion and/or transmembrane transport. This

480 corresponds with previous analyses that found that genes encoding transmembrane proteins

481 tended to be closer to telomeric ends of chromosomes and were more likely to acquire loss-of-

482 function mutations (Bergström et al., 2014). Of the 1601 genes that have premature stop

483 codons in fewer than 20 strains, 284 are involved in catabolic processes (Holm-Bonferroni

484 test/Benjamini Hochberg p-value $<3 e-4)$ and 385 are involved in responses to stimuli $(p$-value $<$

485 6e-5). The number of genes with loss-of-function variants is much greater than the number

486 found in previous studies, likely due to the fact that this dataset has a greater number of strains

487 and much more diversity among strains in regards to factors such as ploidy and isolation origin

488 (Bergström et al., 2014; Jelier et al., 2011). 


\section{Discussion}

Assessing the phenotype of alleles on a species-wide scale is crucial for understanding

491 how quantitative traits vary in a population. Previously developed approaches for experimentally

492 identifying causal variants are conducted through DNA synthesis or mutagenesis, and in many

493 cases do not reflect alleles found in natural populations. We have developed here a high-

494 throughput and low-cost functional approach that can measure the fitness of nearly all alleles

495 present in a population. Specifically in our study, we investigated the function of 228 natural

496 variants of SUL1, a high-affinity sulfate transporter gene, present in the 1,011 S. cerevisiae

497 strain collection. Our assay identified instances of functional, intermediate, and loss-of-function

498 phenotypes. Using this data, as well as gene and whole genome sequencing data, we related

499 SUL1 fitness to its evolutionary history. SUL1 acquired multiple independent instances of loss of

500 function, the majority of which were due to premature stop codons. Other alleles had frameshift,

501 nonsynonymous, and promoter polymorphisms that negatively affected fitness. These multiple

502 independent instances provide evidence that there may be a fitness trade-off for having a loss-

503 of-function SUL1 allele. The strains carrying these loss-of-function alleles were largely isolated

504 from dairy, beer, and sake clades. Because not all loss-of-function polymorphisms were

505 identical in each clade (for instance, there are three different premature stop codons among

506 dairy strains), these events were likely not due to drift but may have a functional benefit instead.

507 We recognize an alternative possible explanation, which is that some strains, including those

508 from dairy environments, have been shown to naturally carry a high burden of loss of function

509 polymorphisms, and SUL1 could simply represent an easily tolerated loss that is recurrent by

510 chance. As shown by previous studies, enriched loss-of-function events in specific populations

511 are thought to arise as a result of genetic drift and play an important role in maintaining genetic

512 variation (Legras et al., 2018; Zorgo et al., 2012).

513 However, there is some evidence that a loss-of-function SUL1 allele may confer a trade-

514 off and be beneficial under particular environments. Prior studies have shown that there are 
515 toxic analogues of sulfate, such as chromate and selenate, that could be transported into the

516 cell through the Sul1 permease (Cherest et al., 1997; Johnson et al., 2016). Several studies

517 have also identified other toxic compounds such as cadmium that affect cell function and growth

518 due to the uptake of sulfate by Sul1 (Zhang et al., 2020). These show instances where having a

519 functional copy of SUL1 would be detrimental and suggest that SUL1 may have some

520 antagonistic pleiotropic effects. This may also explain the lack of gain-of-function alleles in our

521 library, as having a higher-affinity SUL1 may not be beneficial in natural environments. Despite

522 the results from previous studies, many of which investigated the effects of toxic compounds in

523 lab strain backgrounds similar to what we used here, we have been unable to recapitulate these

524 trade-offs.

$525 \quad$ Identifying loss-of-function alleles by searching for premature stop codons is relatively

526 straightforward. Additionally, we found that many of the nonsynonymous polymorphisms were

527 predicted from mutfunc to have a deleterious effect, although many of these predicted

528 deleterious polymorphisms were false positives. Moreover, the effects of polymorphisms in

529 regulatory regions are more challenging to predict computationally. Using natural variation, we

530 have identified instances where a single polymorphism (n.-456G $>A$ ) in a predicted transcription

531 factor-binding site affects fitness of cells under sulfate limitation, a result that was also apparent

532 in our prior promoter mutagenesis study (Rich et al., 2016).

533 Our approach also identifies intermediate phenotypes, many of which in our pool were

534 likely a result of a natural promoter polymorphism that affects expression. For studying variants,

535 it is challenging to identify deleterious mutations in a population, and here we illustrate an

536 example showing the importance of studying both coding and noncoding polymorphisms, as

537 both normal expression and protein structure affect phenotype and thus how selection acts on a

538 population.

539 While some SUL1 alleles have single polymorphisms that can result in a total loss of

540 function, there were also alleles with several nonsynonymous mutations that had wild-type-like 
541 fitness under sulfate limitation. Notable examples include the two SUL1 alleles found across 21

542 unique isolates that had signatures of introgression from S. paradoxus; these alleles had over

54340 amino acid differences, yet functioned normally in the S288C background. These results

544 support our previous findings that SUL1's high affinity has been maintained across S.

545 paradoxus and S. cerevisiae (Sanchez et al., 2017), and the fitness measurements of the

546 introgressed alleles support the idea that these sequences maintain their function even in a new

547 genetic background context. The wide variation in SUL1 function under sulfate limitation is stark,

548 and using these natural variants has provided further evidence for non-neutral evolution.

549 In this study and our prior study, we found no correlation between SUL1 function and its

550 original isolate's growth on sulfate-limited media (Payen et al., in preparation). Again, despite

551 the fact that SUL1 copy number increases in evolution experiments under sulfate limitation, we

552 were surprised to see that fitness of endogenous copies of SUL1 did not necessarily dictate cell

553 performance under sulfate limitation. One possible reason for this observation is that these

554 strains contain functional copies of the SUL1 paralog, SUL2. Despite being a lower functioning

555 sulfate permease compared to SUL1, we found no strains that were homozygous for obvious

556 loss-of-function SUL2 alleles. The alleles of SUL2 and other transporters like SOA1 likely also

557 play an important role in growth under sulfate-limiting conditions. Alternatively, small growth rate

558 changes may not be observable in our solid media growth rate assays compared to what is

559 possible to measure in chemostat culture.

562 hundreds of natural variants in high-throughput. Assaying natural variants in this manner is

563 especially useful when coupled with whole-genome sequencing data, as it allows us to better

564 understand function in relation to molecular evolution. Furthermore, our method compares many

565 alleles of a gene in isolation in an otherwise isogenic background away from the complexities of

566 genetic background interactions. This approach complements methods like QTL mapping, 
567 providing a more thorough investigation of phenotypic patterns across an entire species, which

568 can also contribute to our understanding of how pleiotropic a gene is. Further application of this

569 approach in other genes and other genetic backgrounds will be greatly beneficial to our

570 understanding of how selection acts on natural populations and how multiple polymorphisms

571 contribute to function and ultimately phenotype.

572

573 Acknowledgements

574 The authors thank members of the Dunham lab for their help: M. Bryce Taylor and

575 Abigail Keller for assisting in experimental design and analysis; Clara Amorosi and Christopher

576 Ryan Livingston Large for helping with quality control and providing input on data visualization;

577 and Noah A. Hanson for sequencing samples. We also thank Anne Clark and Josh Akey for

578 helping with the sequence analysis and identification of introgressed alleles. We are grateful for

579 Mary Kuhner for her consultation and expertise on the best approach for creating and

580 interpreting our phylogenetic trees, and for Pengyao Jiang and Kelley Harris for assisting with

581 visualization of these trees. The authors also thank Fangfei Li and Sasha Levy for patiently

582 assisting us with FitSeq in MATLAB. Additionally, we would like to thank the UW PacBio

583 Sequencing Core for sequencing our samples while also helping us with optimizing the quality

584 of our libraries. This work was supported by the National Science Foundation Graduate

585 Research Fellowship (DGE-1762114). Research reported in this publication was supported by

586 the National Institute of General Medical Sciences of the National Institutes of Health under

587 award number R01GM101091. The research of MJD was supported in part by a Faculty Scholar

588 grant from the Howard Hughes Medical Institute.

589

590

591

592 


\section{References}

594 1. Adzhubei IA, Schmidt S, Peshkin L, Ramensky VE, Gerasimova A, Bork P, Kondrashov AS, Sunyaev SR. 2010. A method and server for predicting damaging missense mutations. Nature Methods. doi:10.1038/nmeth0410-248

2. Balakrishnan R, Park J, Karra K, Hitz BC, Binkley G, Hong EL, Sullivan J, Micklem G, Cherry JM. 2012. YeastMine-An integrated data warehouse for Saccharomyces cerevisiae data as a multipurpose tool-kit. Database 2012. doi:10.1093/database/bar062

3. Bergström A, Simpson JT, Salinas F, Barré B, Parts L, Zia A, Nguyen Ba AN, Moses AM, Louis EJ, Mustonen V, Warringer J, Durbin R, Liti G. 2014. A high-definition view of functional genetic variation from natural yeast genomes. Molecular Biology and Evolution 31:872-888. doi:10.1093/molbev/msu037

4. Charlesworth J, Eyre-Walker A. 2008. The McDonald-Kreitman Test and Slightly Deleterious Mutations. Molecular Biology and Evolution 25:1007-1015. doi:10.1093/molbev/msn005

5. Cherest H, Davidian JC, Thomas D, Benes V, Ansorge W, Surdin-Kerjan Y. 1997. Molecular characterization of two high affinity sulfate transporters in Saccharomyces cerevisiae. Genetics 145:627-35. mutation and selection on plasticity of a promoter activity in Saccharomyces cerevisiae. Proceedings of the National Academy of Sciences of the United States of America

614 7. Edgar RC. 2004. MUSCLE: Multiple sequence alignment with high accuracy and high 615 throughput. Nucleic Acids Research 32:1792-1797. doi:10.1093/nar/gkh340

616 8. Ehrenreich IM, Bloom J, Torabi N, Wang X, Jia Y, Kruglyak L. 2012. Genetic architecture of 617 highly complex chemical resistance traits across four yeast strains. PLoS Genetics 8.

618 doi:10.1371/journal.pgen.1002570 
619 9. Ehrenreich IM, Gerke JP, Kruglyak L. 2009. Genetic dissection of complex traits in yeast: Insights from studies of gene expression and other phenotypes in the BYxRM cross. Cold Spring Harbor Symposia on Quantitative Biology. NIH Public Access. pp. 145-153. doi:10.1101/sqb.2009.74.013

10. Felsenstein J. 2005. PHYLIP (Phylogeny Inference Package) version 3.6. Distributed by the author Department of Genome Sciences, University of Washington, Seattle.

11. Fowler DM, Fields S. 2014. Deep mutational scanning: A new style of protein science. Nature Methods. doi:10.1038/nmeth.3027

12. Gresham D, Desai MM, Tucker CM, Jenq HT, Pai DA, Ward A, DeSevo CG, Botstein D,

13. Jelier R, Semple JI, Garcia-Verdugo R, Lehner B. 2011. Predicting phenotypic variation in yeast from individual genome sequences. Nature Genetics 43. doi:10.1038/ng.1007

633 14. Johnson AJ, Veljanoski F, O 'doherty PJ, Zaman MS, Petersingham G, Bailey TD, Mü G, Kersaitis C, Wu MJ. 2016. Revelation of molecular basis for chromium toxicity by phenotypes of Saccharomyces cerevisiae gene deletion mutants. Metallomics 8:542-550. doi:10.1039/c6mt00039h

15. Johnson T, Barton N. 2005. Theoretical models of selection and mutation on quantitative traits. Philosophical Transactions of the Royal Society B: Biological Sciences 360:14111425. doi:10.1098/rstb.2005.1667

16. Kim HS, Huh J, Riles L, Reyes A, Fay JC. 2012. A noncomplementation screen for quantitative trait alleles in Saccharomyces cerevisiae. G3: Genes, Genomes, Genetics 2:753-760. doi:10.1534/g3.112.002550

17. Legras, J.-L., Galeote, V., Bigey, F., Camarasa, C., Marsit, S., Nidelet, T., Sanchez, I., Couloux, A., Guy, J., Franco-Duarte, R., Marcet-Houben, M., Gabaldon, T., Schuller, D., 
Sampaio, J. P., Dequin, S., \& Wittkopp, P. (2018). Adaptation of S. cerevisiae to

Fermented Food Environments Reveals Remarkable Genome Plasticity and the Footprints of Domestication. Molecular Biology and Evolution. https://doi.org/10.1093/molbev/msy066

18. Li F, Salit ML, Levy SF. 2018. Unbiased Fitness Estimation of Pooled Barcode or Amplicon Sequencing Studies. Cell Systems 7:521-525.e4. doi:10.1016/j.cels.2018.09.004

19. Li H. 2013. Aligning sequence reads, clone sequences and assembly contigs with BWAMEM.

20. Li H, Handsaker B, Wysoker A, Fennell T, Ruan J, Homer N, Marth G, Abecasis G, Durbin R. 2009. The Sequence Alignment/Map format and SAMtools. Bioinformatics 25:20782079. doi:10.1093/bioinformatics/btp352

21. Liti G, Carter DM, Moses AM, Warringer J, Parts L, James SA, Davey RP, Roberts IN, Burt

A, Koufopanou V, Tsai IJ, Bergman CM, Bensasson D, O’Kelly MJT, van Oudenaarden A, Barton DBH, Bailes E, Nguyen AN, Jones M, Quail MA, Goodhead I, Sims S, Smith F, Blomberg A, Durbin R, Louis EJ. 2009. Population genomics of domestic and wild yeasts. Nature 458:337-341. doi:10.1038/nature07743 and prospects. Nature Reviews Genetics. doi:10.1038/nrg2612 Khechaduri A, Dines JN, Hause RJ, Bhatia S, Evans WE, Relling M v., Yang W, Shendure J, Fowler DM. 2018. Multiplex assessment of protein variant abundance by massively parallel sequencing. Nature Genetics 1. doi:10.1038/s41588-018-0122-z

24. Mitchell-Olds T, Willis JH, Goldstein DB. 2007. Which evolutionary processes influence natural genetic variation for phenotypic traits? Nature Reviews Genetics 8:845-856. doi:10.1038/nrg2207 
25. Morgante F, Huang W, Maltecca C, Mackay TFC. 2018. Effect of genetic architecture on the prediction accuracy of quantitative traits in samples of unrelated individuals. Heredity 120:500-514. doi:10.1038/s41437-017-0043-0

26. Needleman SB, Wunsch CD. 1970. A general method applicable to the search for similarities in the amino acid sequence of two proteins. Journal of Molecular Biology 48:443-453. doi:10.1016/0022-2836(70)90057-4

27. Payen C, di Rienzi SC, Ong GT, Pogachar JL, Sanchez JC, Sunshine AB, Raghuraman

28. Peltier E, Friedrich A, Schacherer J, Marullo P. 2019. Quantitative trait nucleotides Frontiers in Genetics 10:683. doi:10.3389/fgene.2019.00683

29. Peter J, de Chiara M, Friedrich A, Yue J-X, Pflieger D, Bergström A, Sigwalt A, Barre B, Freel K, Llored A, Cruaud C, Labadie K, Aury J-M, Istace B, Lebrigand K, Barbry P,

30. Rich MS, Payen C, Rubin AF, Ong GT, Sanchez MR, Yachie N, Dunham MJ, Fields S. 2016. Comprehensive analysis of the SUL1 promoter of Saccharomyces cerevisiae. Genetics 203:191-202. doi:10.1534/genetics.116.188037 1,011 Saccharomyces cerevisiae isolates. Nature 556:339-344. doi:10.1038/s41586-018$0030-5$

31. Sanchez MR, Miller AW, Liachko I, Sunshine AB, Lynch B, Huang M, Alcantara E, DeSevo CG, Pai DA, Tucker CM, Hoang ML, Dunham MJ. 2017. Differential paralog divergence modulates genome evolution across yeast species. PLoS Genetics 13. doi:10.1371/journal.pgen.1006585 

survey elucidates population structure of Saccharomyces cerevisiae. Nature 458:342-345. doi:10.1038/nature07670

33. Schymkowitz J, Borg J, Stricher F, Nys R, Rousseau F, Serrano L. 2005. The FoldX web server: An online force field. Nucleic Acids Research 33:W382-W388. doi:10.1093/nar/gki387

34. Sharma AK, Rigby AC, Alper SL. 2011. STAS domain structure and function. Cellular Physiology and Biochemistry. doi:10.1159/000335104

35. She R, Jarosz DF. 2018. Mapping Causal Variants with Single-Nucleotide Resolution Reveals Biochemical Drivers of Phenotypic Change. Cell 172:478-490.e15. doi:10.1016/j.cell.2017.12.015

36. Siggers T, Duyzend MH, Reddy J, Khan S, Bulyk ML. 2011. Non-DNA-binding cofactors enhance DNA-binding specificity of a transcriptional regulatory complex. Molecular Systems Biology 7. doi:10.1038/msb.2011.89 of Human Genetics 101:315-325. doi:10.1016/j.ajhg.2017.07.014

38. Stinchcombe JR, Hoekstra HE. 2008. Combining population genomics and quantitative genetics: Finding the genes underlying ecologically important traits. Heredity. doi:10.1038/sj.hdy.6800937

714 39. Strope PK, Skelly DA, Kozmin SG, Mahadevan G, Stone EA, Magwene PM, Dietrich FS, McCusker JH. 2015. The 100-genomes strains, an S. cerevisiae resource that illuminates its natural phenotypic and genotypic variation and emergence as an opportunistic pathogen. Genome Research 125:762-774. doi:10.1101/gr.185538.114 
40. Treusch S, Albert FW, Bloom JS, Kotenko IE, Kruglyak L. 2015. Genetic Mapping of MAPK-Mediated Complex Traits Across S. cerevisiae. PLoS Genetics 11. doi:10.1371/journal.pgen.1004913

41. Wagih O, Galardini M, Busby BP, Memon D, Typas A, Beltrao P. 2018. A resource of variant effect predictions of single nucleotide variants in model organisms. Molecular Systems Biology 14. doi:10.15252/msb.20188430

42. Wagih O, Parts L. 2014. Gitter: A robust and accurate method for quantification of colony sizes from plate images. G3: Genes, Genomes, Genetics 4:547-552. doi:10.1534/g3.113.009431

43. Weile J, Roth FP. 2018. Multiplexed assays of variant effects contribute to a growing genotype-phenotype atlas. Human Genetics. doi:10.1007/s00439-018-1916-x

44. Wilkening S, Lin G, Fritsch ES, Tekkedil MM, Anders S, Kuehn R, Nguyen M, Aiyar RS, cerevisiae. Genetics 196:853-865. doi:10.1534/genetics.113.160291 predicting complex traits from SNPs. Nature Reviews Genetics. doi:10.1038/nrg3457 and annotation of phylogenetic trees with their covariates and other associated data. Methods in Ecology and Evolution 8:28-36. doi:10.1111/2041-210X.12628

47. Zhang J, Kobert K, Flouri T, Stamatakis A. 2014. PEAR: A fast and accurate Illumina Paired-End reAd mergeR. Bioinformatics 30:614-620. doi:10.1093/bioinformatics/btt593 48. Zhang X, Kuang X, Cao F, Chen R, Fang Z, Liu W, Shi P, Wang H, Shen Y, Huang Z. 2020. Effect of cadmium on mRNA mistranslation in Saccharomyces cerevisiae. Journal of Basic Microbiology. doi:10.1002/jobm.201900495 
743 49. Zhu YO, Sherlock G, Petrov DA. 2016. Whole genome analysis of 132 clinical

744 Saccharomyces cerevisiae strains reveals extensive ploidy variation. G3: Genes,

$745 \quad$ Genomes, Genetics 6:2421-2434. doi:10.1534/g3.116.029397

746 50. Zorgo, E., Gjuvsland, A., Cubillos, F. A., Louis, E. J., Liti, G., Blomberg, A., Omholt, S. W.,

747 \& Warringer, J. (2012). Life History Shapes Trait Heredity by Accumulation of Loss-of-

748 Function Alleles in Yeast. Molecular Biology and Evolution, 29(7), 1781-1789.

749 https://doi.org/10.1093/molbev/mss019 
750 Supplemental Figure 1. Percentage of strains for each ploidy that matched to at least one

751 PacBio read.

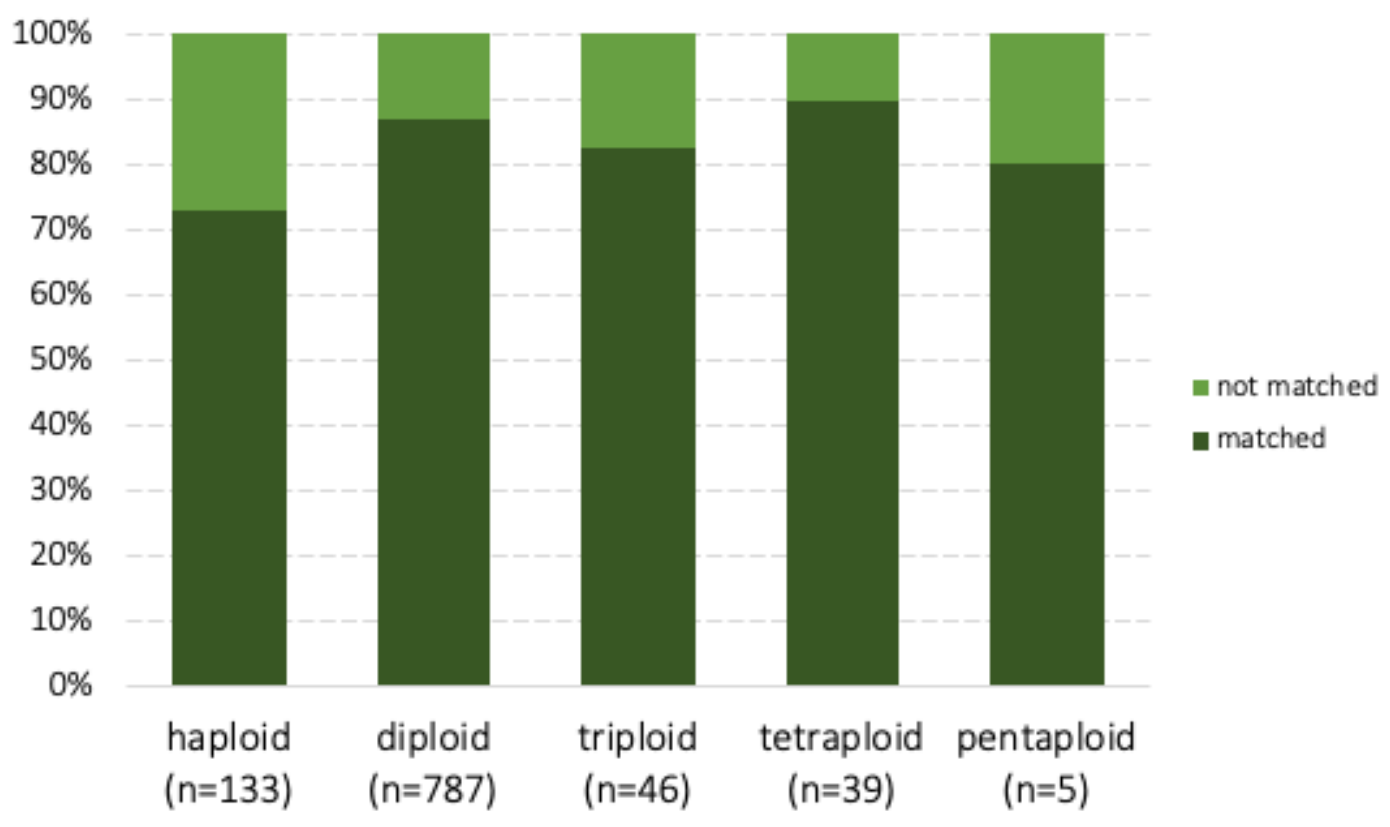

753 Supplemental Figure 2. Allele frequencies found in PacBio allele library reflect those found in

754 the Illumina reference sequences (expected values).

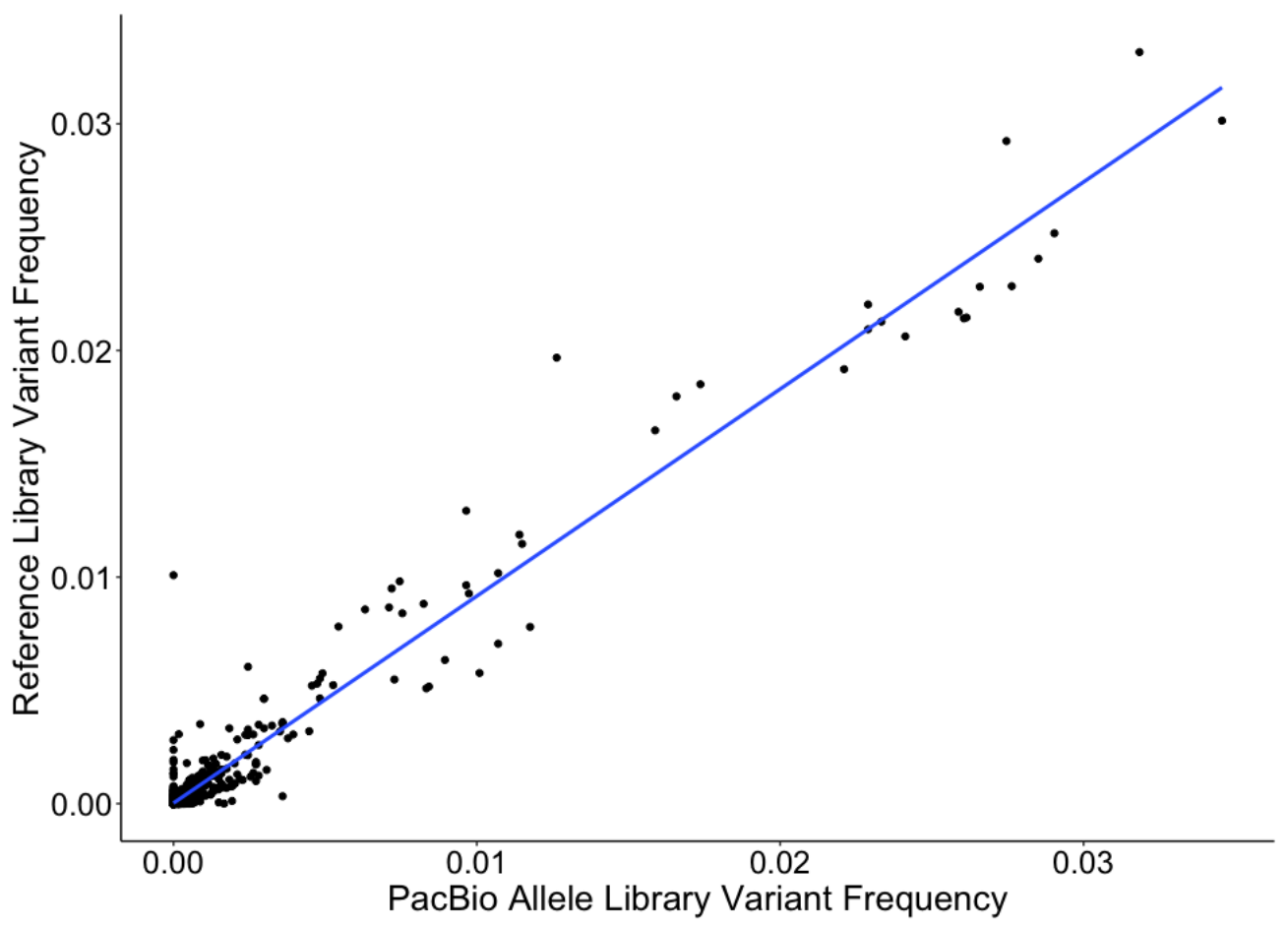


755 Supplemental Figure 3. Competitive fitness values calculated using FitSeq are well-correlated

756 across replicates. Pearson correlation coefficients $r$ are listed on the top half. ${ }^{* * *} p<2.2 e-16$
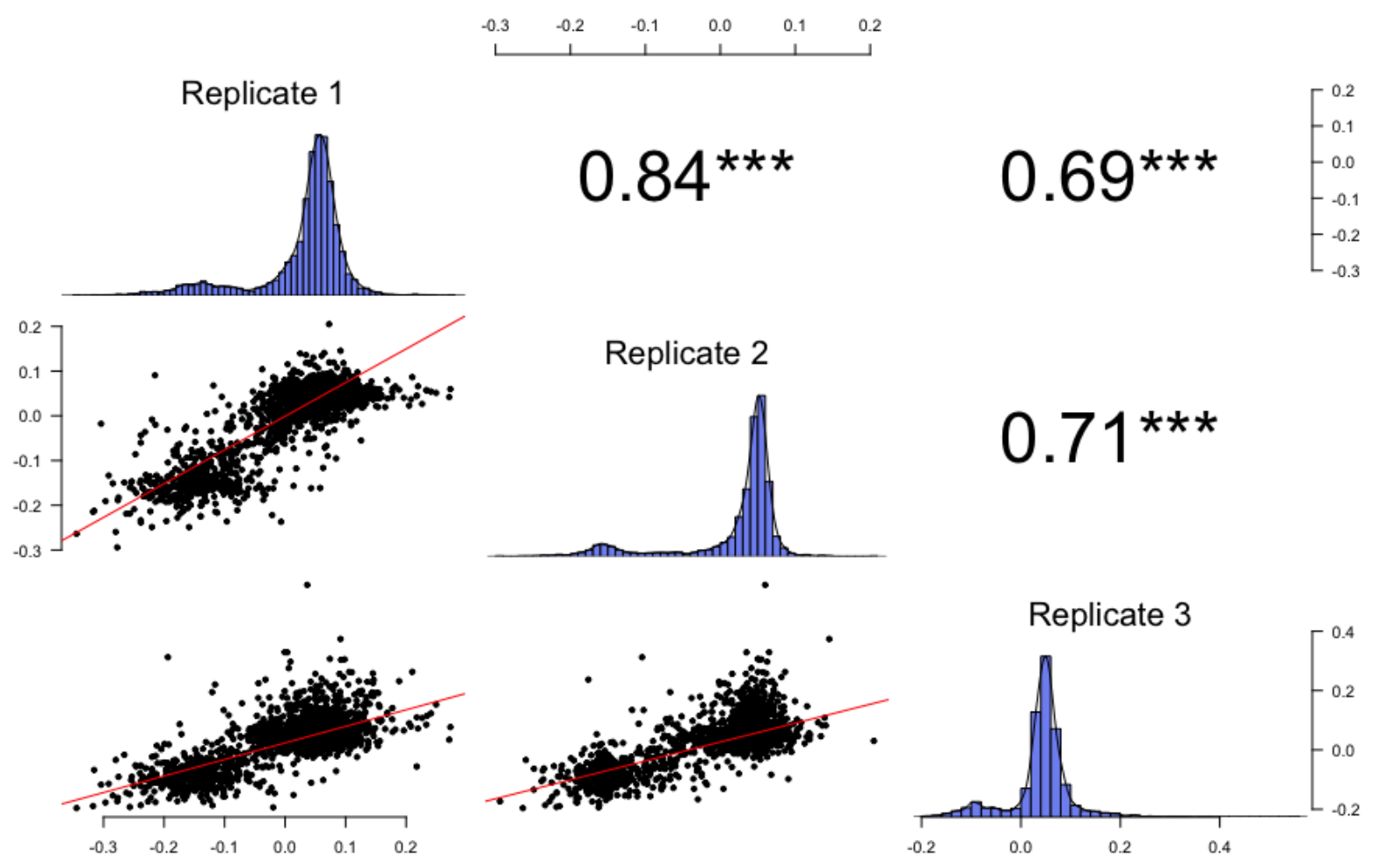
758 Supplemental Figure 4. a) Barplot representing average fitness and standard deviation of

759 barcodes categorized by location of premature stop codons. Sites without error bars are

760 represented by only one barcode. b) Barcodes associated with loss-of-function alleles tend to

761 have greater variance compared to barcodes with wild-type fitness.

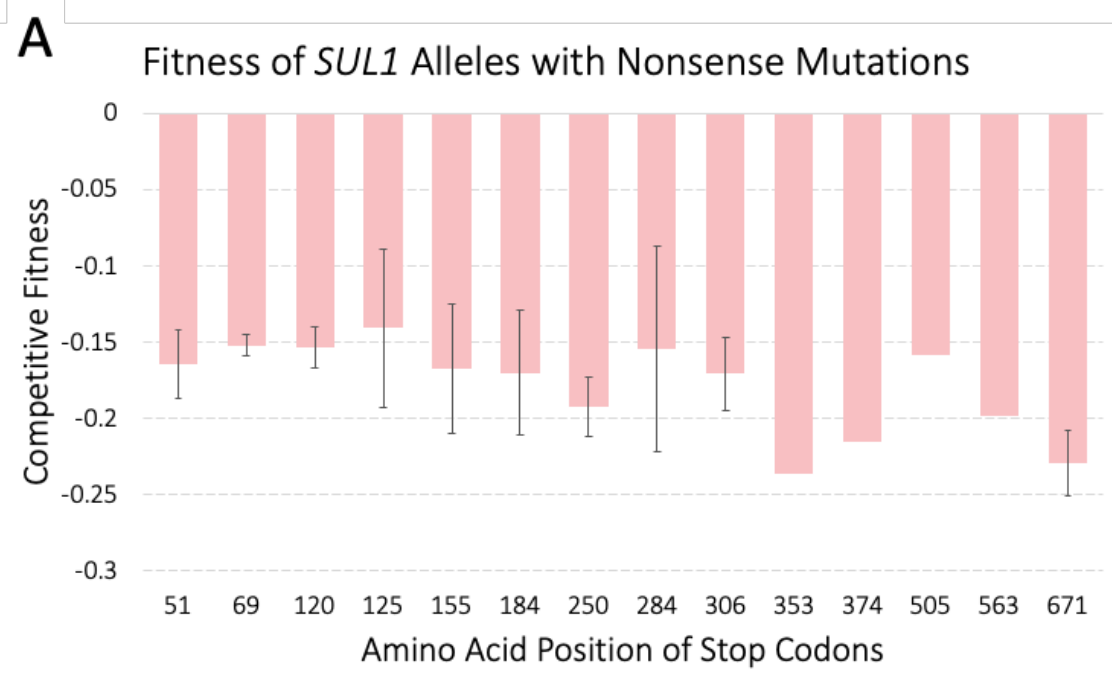

B

Variance Among Barcodes with Identical Alleles

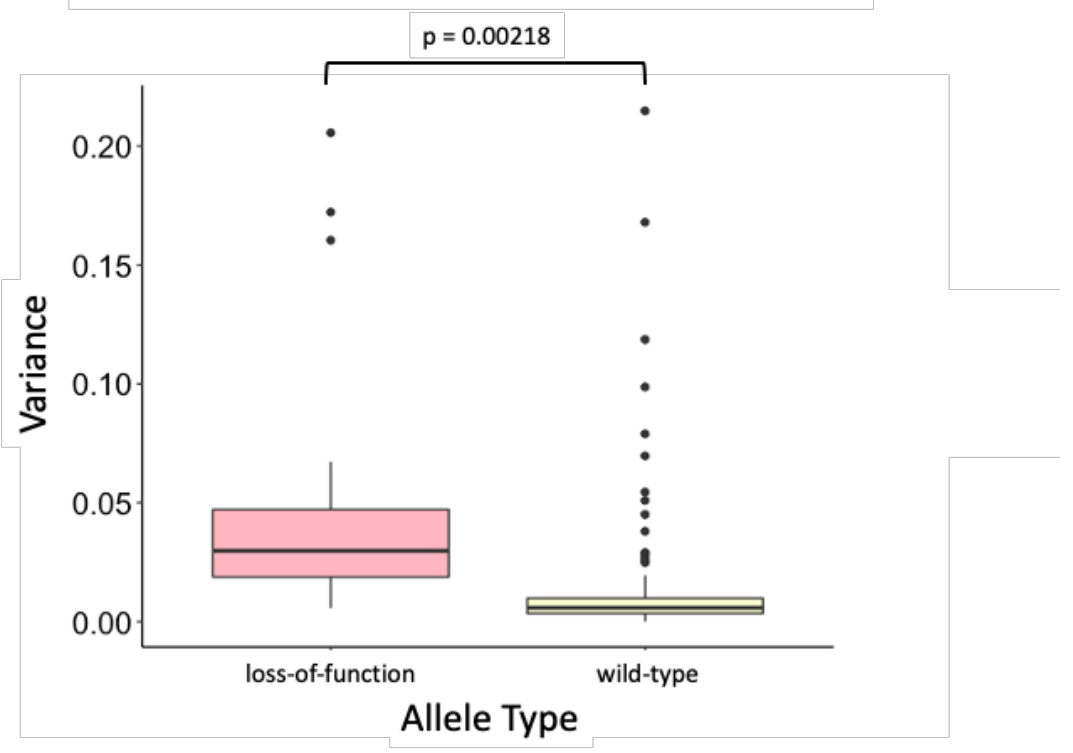


762 Supplemental Figure 5. mutfunc determines which mutations are deleterious. For our data,

763 mutfunc returned SIFT scores for each mutation. We used the mutation with the most

764 deleterious SIFT scores for each allele. a) Competitive fitness of allele from pooled natural

765 variant library plotted against SIFT score of most deleterious mutation shows very little

766 correlation (Pearson's correlation $r=0.253$ ). b) Distribution of experimentally assayed compared

767 with mutfunc predictions of deleteriousness.
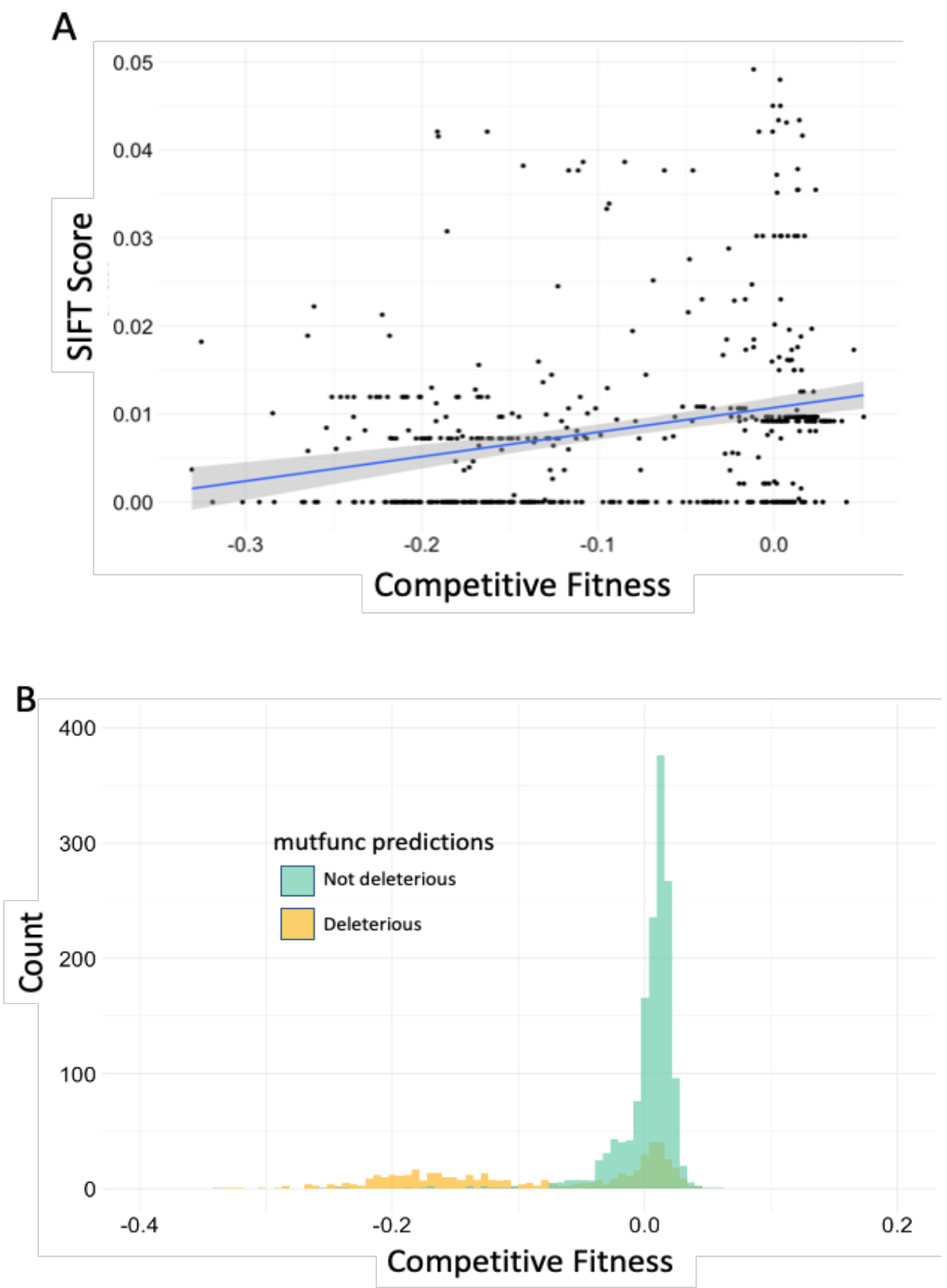
768 Supplemental Figure 6. a) Growth rate of sul1 $\Delta$ sul2 $\Delta$ strains (orange) and wild-type strain

769 (blue) show differential growth on sulfate-limited media. b) Scatterplot comparing strain

770 competitive fitness with growth rate on solid sulfate-limited media show no correlation. c)

771 Grouped by clade, ploidy, zygosity, and continent, strains show no obvious pattern

A

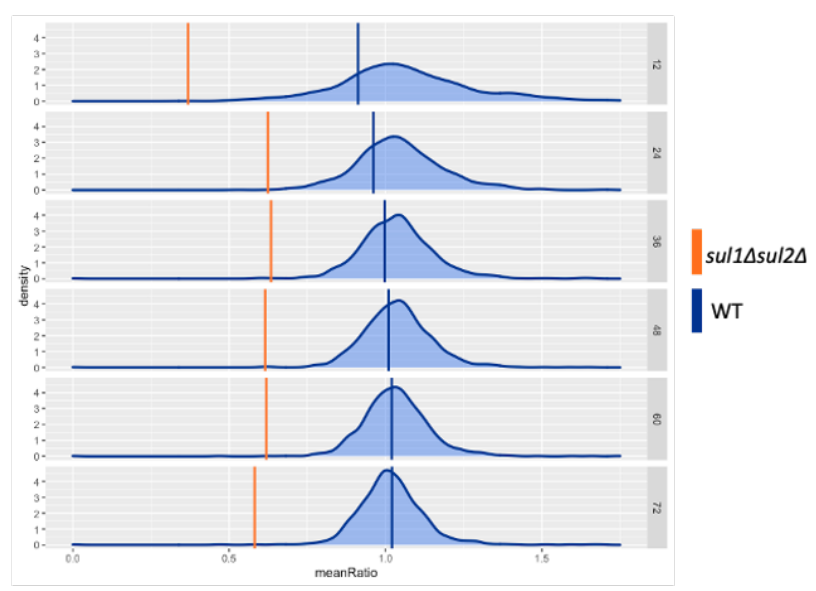

C
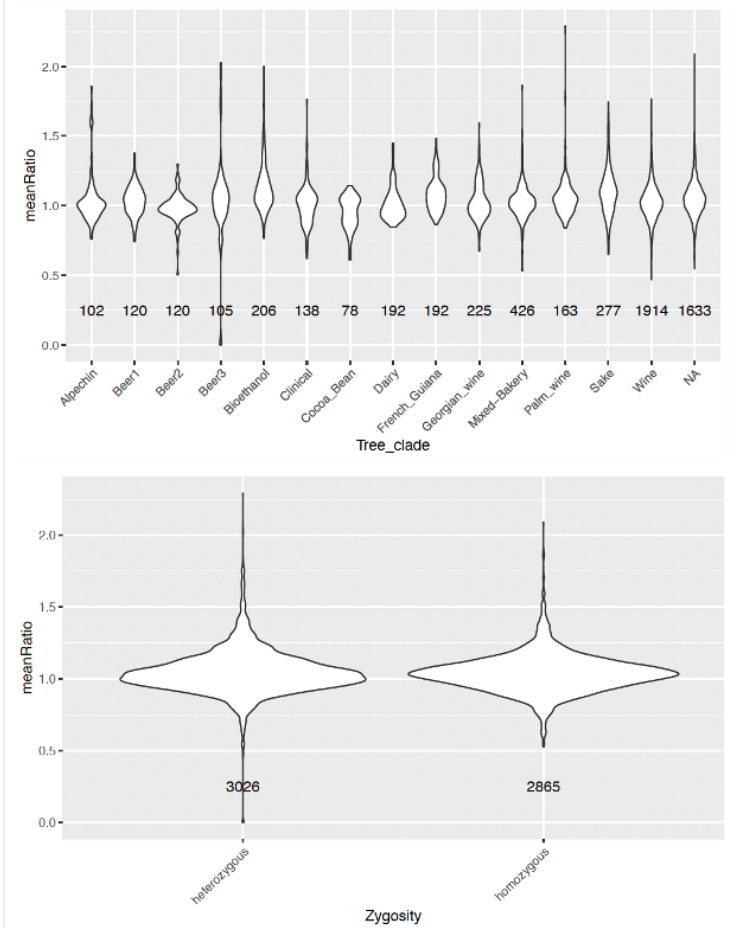

B

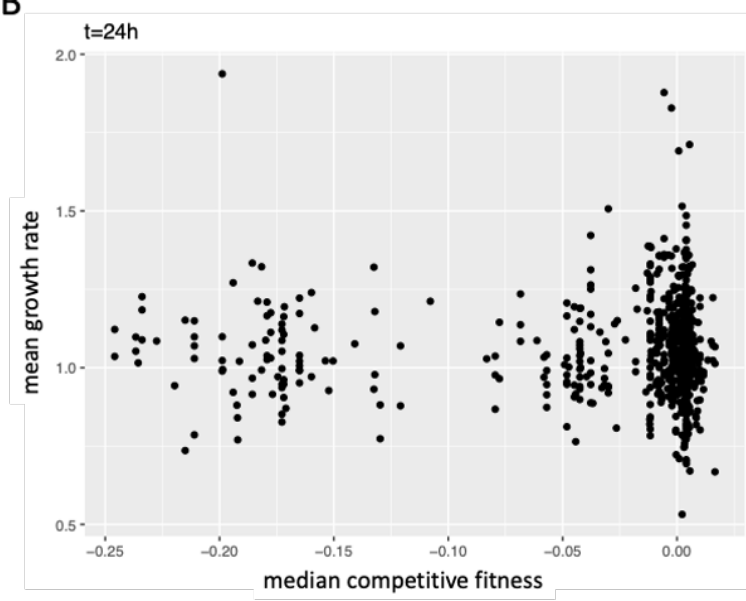

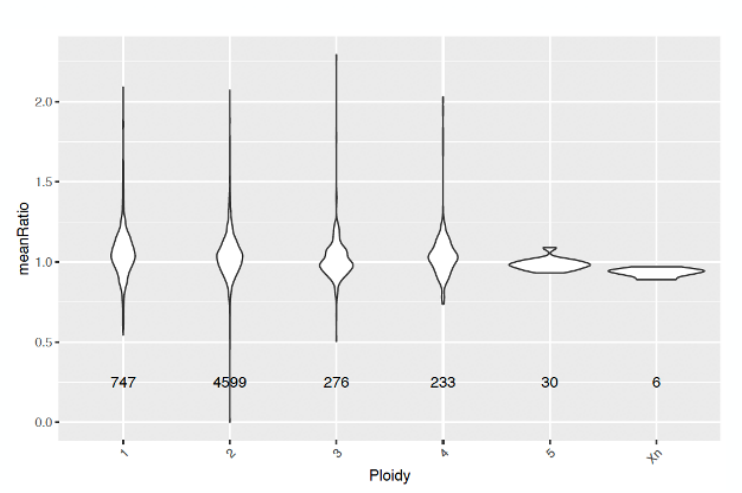

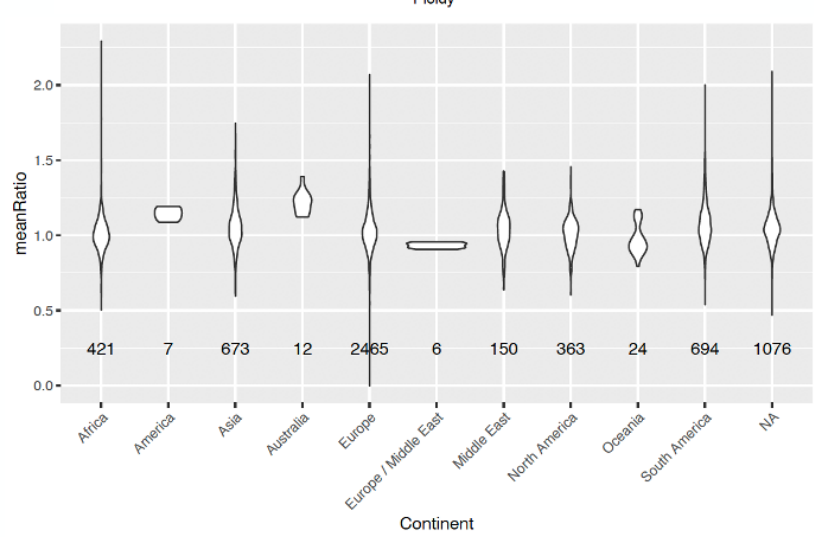


bioRxiv preprint doi: https://doi. org/10.1101/2021.02 26.433108; this version posted February 26, 2021. The copyright holder for this preprint (which was not certified by peer review) is the author/funder, who has granted bioRxiv a license to display the preprint in perpetuity. It is made available under aCC-BY-NC-ND 4.0 International license.

772 Supplemental Figure 7. Barplot of number of genes with premature stop codons per strain,

773 grouped by ecological origins.

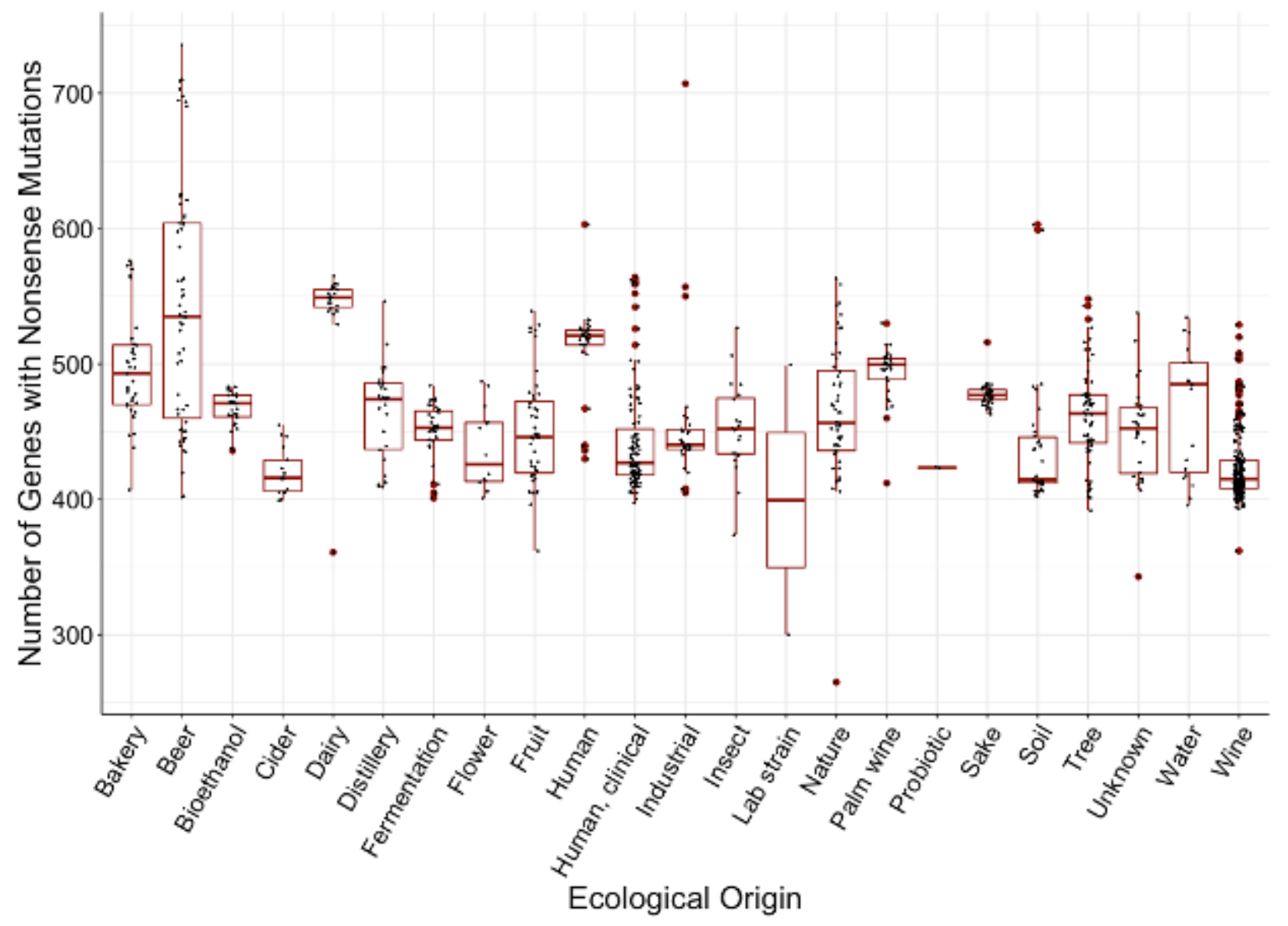

